\title{
Sparse Formulae for the Distance Modulus in Cosmology
}

\author{
Lorenzo Zaninetti \\ Physics Department, Turin, Italy \\ Email: 1.zaninetti@alice.it
}

How to cite this paper: Zaninetti, L. (2021) Sparse Formulae for the Distance Modulus in Cosmology. Journal of High Energy Physics, Gravitation and Cosmology, 7, 965-992.

https://doi.org/10.4236/jhepgc.2021.73057

Received: March 18, 2021

Accepted: July 3, 2021

Published: July 6, 2021

Copyright $\odot 2021$ by author(s) and Scientific Research Publishing Inc. This work is licensed under the Creative Commons Attribution International License (CC BY 4.0).

http://creativecommons.org/licenses/by/4.0/

\section{(c) (i) Open Access}

\begin{abstract}
We review the distance modulus in twelve different cosmologies: the $\Lambda \mathrm{CDM}$ model, the wCDM model, the Cardassian model, the flat case, the $\phi \mathrm{CDM}$ cosmology, the Einstein-De Sitter model, the modified Einstein-De Sitter model, the simple GR model, the flat expanding model, the Milne model, the plasma model and the modified tired light model. The above distance moduli are processed for three different compilations of supernovae and a supernovae + GRBs compilation: Union 2.1, JLA, the Pantheon and Union $2.1+59$ GRBs. For each of the 48 analysed cases we report the relative cosmological parameters, the chi-square, the reduced chi-square, the AIC and the $Q$ parameter. The angular distance as function of the redshift for five cosmologies is reported in the framework of the minimax approximation.
\end{abstract}

\section{Keywords}

Cosmology, Observational Cosmology, Distances, Redshifts, Radial

Velocities, Spatial Distribution of Galaxies, Magnitudes and Colours, Luminosities

\section{Introduction}

At the moment of writing, the determination of the Hubble constant is oscillating between a low value as derived by the Planck collaboration [1],

$H_{0}=(67.4 \pm 0.5) \mathrm{km} \cdot \mathrm{s}^{-1} \cdot \mathrm{Mpc}^{-1}$, and an high value, $H_{0}=(74.03 \pm 1.42) \mathrm{km} \cdot \mathrm{s}^{-1} \cdot \mathrm{Mpc}^{-1}$, as measured on 70 long-period Cepheids in the Large Magellanic Cloud (LMC) [2]. The above difference is referred to as the Hubble constant tension [3] and takes the value of $4.4 \sigma$. It fixes an acceptable interval for the evaluation of $H_{0}$. The number of supernovae (SNs) of type Ia for which the distance modulus is available has grown with time: 34 SNs in the sample which produced evidence for the accelerating universe [4], 580 SNs in the 
Union 2.1 compilation [5], $740 \mathrm{SNs}$ in the joint light-curve analysis (JLA) [6], and 1048 SNs in the Pantheon sample [7] [8]. The availability of SN compilations allows testing old and new cosmological models. We select some of them among others: cosmological relativity in five spatial dimensions [9], an improvement of the Einstein-De Sitter cosmology [10], the $f(R)$ gravity with additional logarithmic corrections [11] [12], influence of the detection of gravitational waves on a definitive theory of gravity [13], the derivation of the value of the Hubble constant as $H_{0}=(70.5 \pm 0.5) \mathrm{km} \cdot \mathrm{s}^{-1} \cdot \mathrm{Mpc}^{-1}$ in the framework of the dark energy cosmology [14] and the deduction of the parameters for Starobinsky gravity [15]. This paper reviews, in Section 2, old and new distance moduli in twelve cosmologies. Then Section 3 processes the analysed cosmologies in four compilations of SNs.

\section{Different Cosmologies}

In the following, we analyze twelve cosmologies. A useful introduction to the distances in cosmology can be found in [16].

\subsection{The Standard Cosmology}

In $\Lambda \mathrm{CDM}$ cosmology the Hubble distance $D_{\mathrm{H}}$ is defined as

$$
D_{\mathrm{H}} \equiv \frac{c}{H_{0}},
$$

where $c$ is the speed of light and $H_{0}$ is the Hubble constant. We then introduce the first parameter $\Omega_{\mathrm{M}}$,

$$
\Omega_{\mathrm{M}}=\frac{8 \pi G \rho_{0}}{3 H_{0}^{2}},
$$

where $G$ is the Newtonian gravitational constant and $\rho_{0}$ is the mass density at the present time. A second parameter is $\Omega_{\Lambda}$,

$$
\Omega_{\Lambda} \equiv \frac{\Lambda c^{2}}{3 H_{0}^{2}}
$$

where $\Lambda$ is the cosmological constant, see [17]. Once $\Omega_{\Lambda}$ and $H_{0}$ are found the numerical value of the cosmological constant is derived, $\Lambda \approx 1.2 \mathrm{~m}^{-2}$.

The two previous parameters are connected with the curvature $\Omega_{K}$ by

$$
\Omega_{\mathrm{M}}+\Omega_{\Lambda}+\Omega_{K}=1 .
$$

The comoving distance, $D_{\mathrm{C}}$, is

$$
D_{\mathrm{C}}=D_{\mathrm{H}} \int_{0}^{z} \frac{\mathrm{d} z^{\prime}}{E\left(z^{\prime}\right)},
$$

where $E(z)$ is the "Hubble function":

$$
E(z)=\sqrt{\Omega_{\mathrm{M}}(1+z)^{3}+\Omega_{K}(1+z)^{2}+\Omega_{\Lambda}} .
$$

The above integral cannot be done in analytical terms, except for the case of $\Omega_{\Lambda}=0$, but the Padé approximant, see Appendix 5, allows to derive the ap- 
proximated indefinite integral, see Equation (10).

The approximate definite integral for (5) is therefore,

$$
D_{\mathrm{C}, 2,2}=D_{\mathrm{H}}\left(F_{2,2}\left(z ; a_{0}, a_{1}, a_{2}, b_{0}, b_{1}, b_{2}\right)-F_{2,2}\left(0 ; a_{0}, a_{1}, a_{2}, b_{0}, b_{1}, b_{2}\right)\right),
$$

where $F_{2,2}$ is Equation (10). The transverse comoving distance $D_{\mathrm{M}}$ is:

$$
D_{\mathrm{M}}= \begin{cases}D_{\mathrm{H}} \frac{1}{\sqrt{\Omega_{K}}} \sinh \left[\sqrt{\Omega_{K}} D_{\mathrm{C}} / D_{\mathrm{H}}\right] & \text { for } \Omega_{K}>0 \\ D_{\mathrm{C}} & \text { for } \Omega_{K}=0 \\ D_{\mathrm{H}} \frac{1}{\sqrt{\left|\Omega_{K}\right|}} \sin \left[\sqrt{\left|\Omega_{K}\right|} D_{\mathrm{C}} / D_{\mathrm{H}}\right] & \text { for } \Omega_{K}<0\end{cases}
$$

and the approximate transverse comoving distance $D_{\mathrm{M}, 2,2}$ computed with the Padé approximant is:

$$
D_{\mathrm{M}, 2,2}= \begin{cases}D_{\mathrm{H}} \frac{1}{\sqrt{\Omega_{K}}} \sinh \left[\sqrt{\Omega_{K}} D_{\mathrm{C}, 2,2} / D_{\mathrm{H}}\right] & \text { for } \Omega_{K}>0 \\ D_{\mathrm{C}, 2,2} & \text { for } \Omega_{K}=0 \\ D_{\mathrm{H}} \frac{1}{\sqrt{\left|\Omega_{K}\right|}} \sin \left[\sqrt{\left|\Omega_{K}\right|} D_{\mathrm{C}, 2,2} / D_{\mathrm{H}}\right] & \text { for } \Omega_{K}<0\end{cases}
$$

The Padé approximant for the luminosity distance is

$$
D_{\mathrm{L}, 2,2}=(1+z) D_{\mathrm{M}, 2,2},
$$

and the Padé approximant for the distance modulus, $(m-M)_{2,2}$, is

$$
(m-M)_{2,2}=25+5 \log _{10}\left(D_{\mathrm{L}, 2,2}\right) \text {. }
$$

As a consequence, $M_{2,2}$, the absolute magnitude of the Padé approximant, is

$$
M_{2,2}=m-25-5 \log _{10}\left(D_{\mathrm{L}, 2,2}\right) \text {. }
$$

The expanded version of the Padé approximant distance modulus is:

$$
(m-M)_{2,2}=25+5 \frac{1}{\ln (10)} \ln \left(\frac{c(1+z)}{H_{0} \sqrt{\Omega_{K}}} \sinh \left(1 / 2 \frac{\sqrt{\Omega_{K}} A}{b_{2}^{2} \sqrt{4 b_{0} b_{2}-b_{1}^{2}}}\right)\right),
$$

with

$$
\begin{aligned}
A= & \ln \left(z^{2} b_{2}+z b_{1}+b_{0}\right) a_{1} b_{2} \sqrt{4 b_{0} b_{2}-b_{1}^{2}}-\ln \left(z^{2} b_{2}+z b_{1}+b_{0}\right) a_{2} b_{1} \sqrt{4 b_{0} b_{2}-b_{1}^{2}} \\
& -\ln \left(b_{0}\right) a_{1} b_{2} \sqrt{4 b_{0} b_{2}-b_{1}^{2}}+\ln \left(b_{0}\right) a_{2} b_{1} \sqrt{4 b_{0} b_{2}-b_{1}^{2}}+2 a_{2} z b_{2} \sqrt{4 b_{0} b_{2}-b_{1}^{2}} \\
& +4 \arctan \left(\frac{2 z b_{2}+b_{1}}{\sqrt{4 b_{0} b_{2}-b_{1}^{2}}}\right) a_{0} b_{2}^{2}-2 \arctan \left(\frac{2 z b_{2}+b_{1}}{\sqrt{4 b_{0} b_{2}-b_{1}^{2}}}\right) b_{1} a_{1} b_{2} \\
& -4 \arctan \left(\frac{2 z b_{2}+b_{1}}{\sqrt{4 b_{0} b_{2}-b_{1}^{2}}}\right) a_{2} b_{0} b_{2}+2 \arctan \left(\frac{2 z b_{2}+b_{1}}{\sqrt{4 b_{0} b_{2}-b_{1}^{2}}}\right) b_{1}^{2} a_{2} \\
& -4 \arctan \left(\frac{b_{1}}{\sqrt{4 b_{0} b_{2}-b_{1}^{2}}}\right) a_{0} b_{2}^{2}+2 \arctan \left(\frac{b_{1}}{\sqrt{4 b_{0} b_{2}-b_{1}^{2}}}\right) b_{1} a_{1} b_{2} \\
& +4 \arctan \left(\frac{b_{1}}{\sqrt{4 b_{0} b_{2}-b_{1}^{2}}}\right) a_{2} b_{0} b_{2}-2 \arctan \left(\frac{b_{1}}{\sqrt{4 b_{0} b_{2}-b_{1}^{2}}}\right) b_{1}^{2} a_{2}
\end{aligned}
$$


Figure 1 reports the percentage error, see formula (75), for $(m-M)_{2,2}$ as function of the redshift until the value of $1 \%$ is reached at $z \approx 6$. For $z>6$ the Padé approximant of the distance modulus does not converge to the numerical distance modulus.

More details can be found in [18].

\subsection{Dynamical Dark Energy or wCDM}

In the dynamical dark energy cosmology (wCDM), firstly introduced by [19], the Hubble distance is

$$
D_{H}\left(z ; \Omega_{\mathrm{M}}, w, \Omega_{D E}\right)=\frac{1}{\sqrt{(1+z)^{3} \Omega_{\mathrm{M}}+\Omega_{D E}(1+z)^{3+3 w}}},
$$

where $w$ is the equation of state here considered constant, see Equation (3.4) in [20] or Equation (18) in [21] for the luminosity distance. Here we considered $W$ to be constant but also the case of $w$ as function of $z$ can be considered, see Equation (19) in [21]. In the above cosmology the cosmological constant is absent. In flat cosmology,

$$
\Omega_{\mathrm{M}}+\Omega_{D E}=1,
$$

and the Hubble distance becomes

$$
D_{H}\left(z ; \Omega_{\mathrm{M}}, w\right)=\frac{1}{\sqrt{(1+z)^{3} \Omega_{\mathrm{M}}+\left(1-\Omega_{\mathrm{M}}\right)(1+z)^{3+3 w}}} .
$$

The indefinite integral in the variable $z$ of the above Hubble distance, $I z \equiv \frac{D_{C}}{D_{H}}$, is

$$
I z\left(z ; \Omega_{\mathrm{M}}, w\right)=\int D_{H}\left(z ; \Omega_{\mathrm{M}}, w\right) \mathrm{d} z,
$$

where the new symbol $I z$ underline the mathematical operation of integration. In order to solve for the indefinite integral we perform a change of variable $1+z=t^{1 / 3}$.

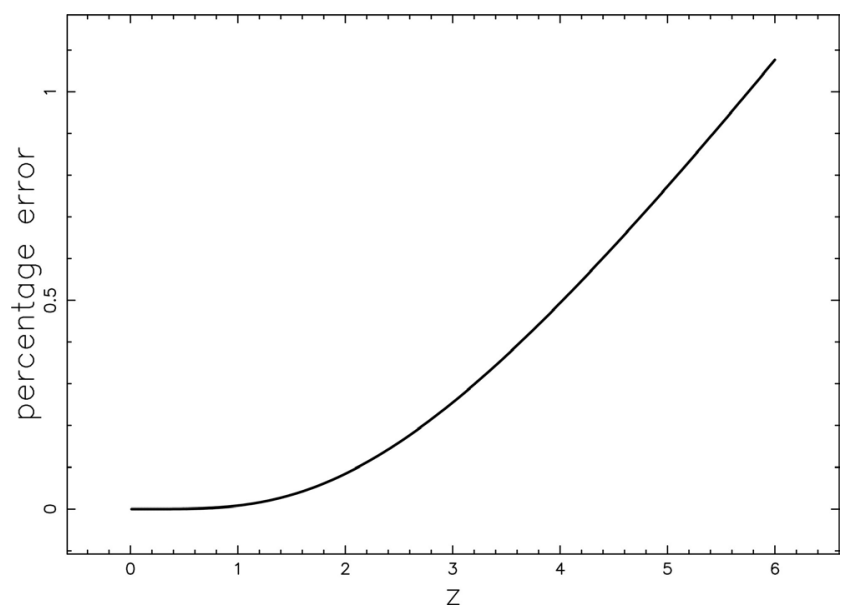

Figure 1. Percentage error of $(m-M)_{2,2}$ in respect to the numerical value with data as in Table 1. 


$$
I z\left(t ; \Omega_{\mathrm{M}}, w\right)=\frac{1}{3} \int \frac{1}{\sqrt{-t\left(\left(-1+\Omega_{\mathrm{M}}\right) t^{w}-\Omega_{\mathrm{M}}\right)} t^{2 / 3}} \mathrm{~d} t .
$$

The indefinite integral is

$$
I z\left(t ; \Omega_{\mathrm{M}}, w\right)=\frac{-2{ }_{2} F_{1}\left(\frac{1}{2},-\frac{1}{6} w^{-1} ; 1-\frac{1}{6} w^{-1} ;-\frac{t^{w}-\left(1-\Omega_{\mathrm{M}}\right)}{\Omega_{\mathrm{M}}}\right)}{\sqrt{\Omega_{\mathrm{M}}} \sqrt[6]{t}},
$$

where ${ }_{2} F_{1}(a, b ; c ; z)$ is the regularized hypergeometric function, see [22] [23] [24] [25] [26]. We now return to the variable $z$, the redshift. Then the indefinite integral becomes:

$$
\begin{aligned}
& \operatorname{Iz}\left(z ; \Omega_{\mathrm{M}}, w\right) \\
& =\frac{-2{ }_{2} F_{1}\left(\frac{1}{2},-\frac{1}{6} w^{-1} ; 1-\frac{1}{6} w^{-1} ;-\frac{\left(-z^{3}+3 z^{2}+3 z+1\right)^{w}\left(1-\Omega_{\mathrm{M}}\right)}{-\Omega_{\mathrm{M}}}\right)}{\sqrt{\Omega_{\mathrm{M}}} \sqrt[6]{z^{3}+3 z^{2}+3 z+1}} .
\end{aligned}
$$

We denote by $F\left(z ; \Omega_{\mathrm{M}}, w\right)$ the definite integral,

$$
F\left(z ; \Omega_{\mathrm{M}}, w\right)=I z\left(z=z ; \Omega_{\mathrm{M}}, w\right)-I z\left(z=0 ; \Omega_{\mathrm{M}}, w\right) .
$$

The luminosity distance, $D_{\mathrm{L}}$, for wCDM cosmology in the case of the analytical solution is

$$
D_{\mathrm{L}}\left(z ; c, H_{0}, \Omega_{\mathrm{M}}, w\right)=\frac{c}{H_{0}}(1+z) F\left(z ; \Omega_{\mathrm{M}}, w\right),
$$

where $F\left(z ; \Omega_{\mathrm{M}}, w\right)$ is given by Equation (21) and the distance modulus is

$$
(m-M)=25+5 \log _{10}\left(D_{\mathrm{L}}\left(z ; c, H_{0}, \Omega_{\mathrm{M}}, w\right)\right) .
$$

More details can be found in [27].

\subsection{The Cardassian Cosmology}

In flat Cardassian cosmology [28] [29] the Hubble distance is

$$
D_{H}\left(z ; \Omega_{\mathrm{M}}, w, n\right)=\frac{1}{\sqrt{(1+z)^{3} \Omega_{\mathrm{M}}+\left(1-\Omega_{\mathrm{M}}\right)(1+z)^{3 n}}},
$$

where $n$ is a variable parameter, and $n=0$ means the $\Lambda$ CDM cosmology, see Equation (17) in [21]. The above equation can also be obtained inserting $n=1+w$ in Equation (14). Despite of this fact the FORTRAN code which derives the cosmological parameters produces a small difference in the results because the variables are evaluated in a different way. The indefinite integral in the variable $z$ of the above Hubble distance, $I z$, is

$$
\operatorname{Iz}\left(z ; \Omega_{\mathrm{M}}, n\right)=\int D_{H}\left(z ; \Omega_{\mathrm{M}}, n\right) \mathrm{d} z .
$$

In order to obtain the indefinite integral we perform a change of variable $1+z=t^{1 / 3}$,

$$
I z\left(t ; \Omega_{\mathrm{M}}, n\right)=\frac{1}{3} \int \frac{1}{\sqrt{-t^{n} \Omega_{\mathrm{M}}+\Omega_{\mathrm{M}} t+t^{n}} t^{2 / 3}} \mathrm{~d} t .
$$


The indefinite integral is

$$
I z\left(t ; \Omega_{\mathrm{M}}, n\right)=\frac{-2{ }_{2} F_{1}\left(1 / 2,-(6 n-6)^{-1} ; \frac{6 n-7}{6 n-6} ; \frac{t^{n-1}\left(\Omega_{\mathrm{M}}-1\right)}{\Omega_{\mathrm{M}}}\right)}{\sqrt{\Omega_{\mathrm{M}}} \sqrt[6]{t}},
$$

where ${ }_{2} F_{1}(a, b ; c ; z)$ is the regularized hypergeometric function. We now return to the original variable $z$ and the indefinite integral is

$$
I z\left(z ; \Omega_{\mathrm{M}}, n\right)=\frac{-2{ }_{2} F_{1}\left(1 / 2,-(6 n-6)^{-1} ; \frac{6 n-7}{6 n-6} ; \frac{\left((1+z)^{3}\right)^{n-1}\left(\Omega_{\mathrm{M}}-1\right)}{\Omega_{\mathrm{M}}}\right)}{\sqrt{\Omega_{\mathrm{M}}} \sqrt[6]{(1+z)^{3}}} .
$$

We denote by $F_{c}\left(z ; \Omega_{\mathrm{M}}, n\right)$ the definite integral,

$$
F_{c}\left(z ; \Omega_{\mathrm{M}}, n\right)=I z\left(z=z ; \Omega_{\mathrm{M}}, n\right)-I z\left(z=0 ; \Omega_{\mathrm{M}}, n\right) .
$$

In the case of the Cardassian cosmology, the luminosity distance is

$$
D_{\mathrm{L}}\left(z ; c, H_{0}, \Omega_{\mathrm{M}}, n\right)=\frac{c}{H_{0}}(1+z) F_{c}\left(z ; \Omega_{\mathrm{M}}, n\right),
$$

where $F_{c}\left(z ; \Omega_{\mathrm{M}}, n\right)$ is given by Equation (29) and the distance modulus is

$$
(m-M)=25+5 \log _{10}\left(D_{\mathrm{L}}\left(z ; c, H_{0}, \Omega_{\mathrm{M}}, n\right)\right) .
$$

In the flat Cardassian cosmology, there are three parameters: $H_{0}, \Omega_{\mathrm{M}}$ and $n$. More details can be found in [27].

\subsection{The Flat Cosmology}

The starting point is Equation (1) for the luminosity distance in [30].

$$
D_{\mathrm{L}}\left(z ; c, H_{0}, \Omega_{\mathrm{M}}\right)=\frac{c(1+z)}{H_{0}} \int_{0}^{z} \frac{1}{\sqrt{\Omega_{\mathrm{M}}(1+t)^{3}+1-\Omega_{\mathrm{M}}}} \mathrm{d} t,
$$

where the variable of integration, $t$, denotes the redshift.

A first change in the parameter $\Omega_{\mathrm{M}}$ introduces

$$
s=\sqrt[3]{\frac{1-\Omega_{\mathrm{M}}}{\Omega_{\mathrm{M}}}}
$$

and the luminosity distance becomes

$$
D_{\mathrm{L}}\left(z ; c, H_{0}, s\right)=\frac{1}{H_{0}} c(1+z) \int_{0}^{z} \frac{1}{\sqrt{\frac{(1+t)^{3}}{s^{3}+1}+1-\left(s^{3}+1\right)^{-1}}} \mathrm{~d} t .
$$

The following change of variable, $t=\frac{s-u}{u}$, is performed for the luminosity distance, which becomes

$$
D_{\mathrm{L}}\left(z ; c, H_{0}, s\right)=-\frac{c}{H_{0} s^{2}}(1+z)\left(s^{3}+1\right) \int_{s}^{\frac{s}{1+z}} \frac{u}{u^{3}+1} \sqrt{\frac{s^{3}\left(u^{3}+1\right)}{u^{3}\left(s^{3}+1\right)}} \mathrm{d} u .
$$


The integral for the luminosity distance is

$$
\begin{aligned}
D_{\mathrm{L}}\left(z ; c, H_{0}, s\right)= & -1 / 3 \frac{c(1+z) 3^{3 / 4} \sqrt{s^{3}+1}}{\sqrt{s} H_{0}} \\
& \times\left(F\left(2 \frac{\sqrt{s(s+1+z)} \frac{4}{3}}{s \sqrt{3}+s+z+1}, 1 / 4 \sqrt{3}+1 / 4 \sqrt{2}\right)\right. \\
& \left.-F\left(2 \frac{\sqrt[4]{3} \sqrt{s(s+1)}}{s+1+s \sqrt{3}}, 1 / 4 \sqrt{2} \sqrt{3}+1 / 4 \sqrt{2}\right)\right),
\end{aligned}
$$

where $s$ is given by Equation (33) and $F(\phi, k)$ is Legendre's incomplete elliptic integral of the first kind,

$$
F(\phi, k)=\int_{0}^{\sin \phi} \frac{\mathrm{d} t}{\sqrt{1-t^{2}} \sqrt{1-k^{2} t^{2}}},
$$

see [26]. The distance modulus is

$$
(m-M)=25+5 \log _{10}\left(D_{\mathrm{L}}\left(z ; c, H_{0}, s\right)\right),
$$

and therefore,

$$
(m-M)=25+5 \frac{1}{\ln (10)} \ln \left(-\frac{1}{3} \frac{c(1+z) 3^{3 / 4}\left(F_{1}-F_{2}\right) \sqrt{s^{3}+1}}{\sqrt{s} H_{0}}\right),
$$

where,

$$
F_{1}=F\left(2 \frac{\sqrt{s(s+1+z)} \sqrt[4]{3}}{s \sqrt{3}+s+z+1}, 1 / 4 \sqrt{2} \sqrt{3}+1 / 4 \sqrt{2}\right)
$$

and

$$
F_{2}=F\left(2 \frac{\sqrt[4]{3} \sqrt{s(s+1)}}{s+1+s \sqrt{3}}, 1 / 4 \sqrt{2} \sqrt{3}+1 / 4 \sqrt{2}\right),
$$

with $s$ as defined by Equation (33). More details can be found in [31].

\section{5. $\phi$ CDM Cosmology}

The inflationary universe has been introduced by [32] [33] [34] and the term "quintessence" in a title of a paper appeared in [35]. At the moment of writing given a scalar field, $\phi$, and the connected self-interacting potential, $V(\phi)$, ten different quintessence models are suggested by [36]. Here we start from Equation (12) in [37] where $E(z)$, the "Hubble function", is

$$
E\left(z ; \Omega_{\mathrm{M} 0}, \Omega_{\mathrm{f} 0} \alpha \beta\right)=\sqrt{(1+z)^{3} \Omega_{\mathrm{M} 0}+\Omega_{\mathrm{f} 0}(1+z)^{\alpha} \mathrm{e}^{\beta z}},
$$

where $\Omega_{\mathrm{M} 0}=\frac{\rho_{m 0}}{3 H_{0}^{2}}$ is the adimensional present density of matter, $\Omega_{\mathrm{f} 0}=\frac{\rho_{\phi 0}}{3 H_{0}^{2}}$ is the present adimensional density of the scalar field, $H_{0}$ is the present value of the Hubble constant, $\rho_{m 0}$ is the present density of matter, $\rho_{\phi 0}$ is the present density of the scalar field, $\alpha$ and $\beta$ are two parameters which allow 
to match theory and observations. In absence of curvature we have

$$
\Omega_{\mathrm{M} 0}+\Omega_{\mathrm{f} 0}=1 \text {, }
$$

and therefore,

$$
E\left(z ; \Omega_{\mathrm{M} 0}, \alpha, \beta\right)=\sqrt{(1+z)^{3} \Omega_{\mathrm{M} 0}+\left(1-\Omega_{\mathrm{M} 0}\right)(1+z)^{\alpha} \mathrm{e}^{\beta z}} .
$$

The luminosity distance is

$$
D_{\mathrm{L}}\left(z ; c, H_{0}, \Omega_{\mathrm{M} 0}, \alpha, \beta\right)=\frac{c(1+z)}{H_{0}} \int_{0}^{z} \frac{1}{E\left(t ; \Omega_{\mathrm{M} 0}, \alpha, \beta\right)} \mathrm{d} t,
$$

where the variable of integration, $t$, denotes the redshift. At the moment of writing there is not an analytical solution for the above integral and therefore we implement a numerical solution, $D_{\mathrm{L}, \text { num }}\left(z ; c, H_{0}, \Omega_{\mathrm{M} 0}, \alpha, \beta\right)$. The distance modulus is

$$
(m-M)=25+5 \log _{10}\left(D_{\mathrm{L}, \text { num }}\left(z ; c, H_{0}, \Omega_{\mathrm{M} 0}, \alpha, \beta\right)\right) .
$$

An approximate value of the above integral (45) is obtained with a Taylor expansion of the integrand about $z=1$ of order seven denoted by $D_{\mathrm{L}, 7}\left(z ; c, H_{0}, \Omega_{\mathrm{M} 0}, \alpha, \beta\right)$. We report the numerical expression with cosmological parameters as in Table 1 relative to the Union 2.1 compilation:

$$
\begin{aligned}
D_{\mathrm{L}, 7}(z)= & 4282.7(1+z)\left(0.91287 z-0.16562 z^{2}+0.039001(z-1)^{3}\right. \\
& -0.003084(z-1)^{4}-0.0036858(z-1)^{5}+0.0028217(z-1)^{6} \\
& \left.-0.00115816(z-1)^{7}+0.03442\right) .
\end{aligned}
$$

The approximate distance modulus is

$$
(m-M)_{7}=25+5 \log _{10}\left(D_{\mathrm{L}, 7}\left(z ; c, H_{0}, \Omega_{\mathrm{M} 0}, \alpha, \beta\right)\right),
$$

which for the Union 2.1 compilation has the following numerical expression,

$$
\begin{aligned}
(m-M)_{7}= & 25+\frac{5}{\ln (10)}\left(\operatorname { l n } \left(4 2 8 2 . 7 ( 1 + z ) \left(0.91287 z-0.16562 z^{2}\right.\right.\right. \\
& +0.039001(z-1)^{3}-0.0030847(z-1)^{4}-0.0036858(z-1)^{5} \\
& \left.\left.\left.+0.0028217(z-1)^{6}-0.0011581(z-1)^{7}+0.03442\right)\right)\right) .
\end{aligned}
$$

Figure 2 reports the percentage error, see formula (75), for $(m-M)_{7}$ as function of the redshift until the value of $0.02 \%$ is reached at $z \approx 2.5$.

\subsection{The Einstein-De Sitter Cosmology}

In the Einstein-De Sitter model the luminosity distance, $D_{L}$, after [38] [39], is

$$
D_{L}=2 \frac{c(1+z-\sqrt{z+1})}{H_{0}},
$$

and the distance modulus for the Einstein-De Sitter model is:

$$
m-M=25+5 \frac{1}{\ln (10)} \ln \left(2 \frac{c(1+z-\sqrt{z+1})}{H_{0}}\right) .
$$




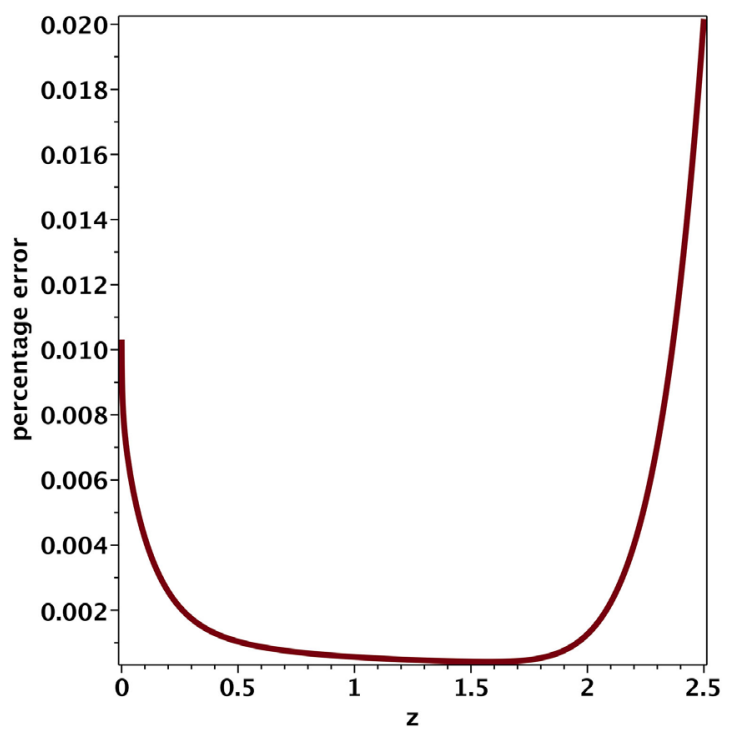

Figure 2. Percentage error of $(m-M)_{7}$ in respect to the numerical value with data as in Table 1.

There is one free parameter in the Einstein-De Sitter model: $H_{0}$. The Einstein-De Sitter model has been recently improved by [10], splitting the analysis in two: the Einstein-De Sitter flat, only-matter universe, referred to as EdesNa, and a flat, only-matter, including the Mach effect universe, referred to as EDSM. We limit ourselves to the EdesNA model and we start from Equation (37) of [10],

$$
m-M=5 \frac{\ln \left(5 / 3 R_{0}(1+z) I_{G}(z)\right)}{\ln (10)}+25,
$$

where,

$$
R_{0}=\frac{c}{H_{0}},
$$

and

$$
I_{G}(z)=\int_{0}^{z} \frac{1}{1+\frac{2}{3}(1+x)^{\frac{3}{2}}} \mathrm{~d} x .
$$

Evaluating the integral yields:

$$
\begin{aligned}
I_{G}(z)= & -\sqrt[6]{3}\left(\arctan \left(\frac{2 \sqrt[6]{3} \sqrt[3]{2}}{3}-\frac{\sqrt{3}}{3}\right)\right. \\
& \left.-\arctan \left(\frac{2 \sqrt[6]{3} \sqrt[3]{2}}{3} \sqrt[3]{(1+z)^{\frac{3}{2}}}-\frac{\sqrt{3}}{3}\right)\right) \sqrt[3]{2}-\frac{12^{\frac{2}{3}}}{12}\left(\ln \left(-\sqrt[3]{2} \sqrt[3]{3}+2^{\frac{2}{3}}+3^{\frac{2}{3}}\right)\right. \\
& -2 \ln (\sqrt[3]{2}+\sqrt[3]{3})+2 \ln \left(\sqrt[3]{2} 3^{2 / 3} \sqrt[3]{(1+z)^{3 / 2}}+3\right) \\
& \left.-\ln \left(2^{\frac{2}{3}} \sqrt[3]{3}\left((1+z)^{\frac{3}{2}}\right)^{\frac{2}{3}}-\sqrt[3]{2} 3^{\frac{2}{3}} \sqrt[3]{(1+z)^{\frac{3}{2}}}+3\right)-\ln (3)\right) .
\end{aligned}
$$


The integrand of (54) can be approximated with a Padé approximant with $p=2, q=2$,

$$
I_{G 22}(z)=\int_{0}^{z} \frac{-3 x^{2}+36 x+144}{67 x^{2}+204 x+240} \mathrm{~d} x,
$$

and therefore we have the approximate integral,

$$
\begin{aligned}
I_{G 22}(z)= & -\frac{3 z}{67}+\frac{1512 \ln \left(67 z^{2}+204 z+240\right)}{4489} \\
& +\frac{64368 \sqrt{1419}}{2123297} \arctan \left(\frac{(134 z+204) \sqrt{1419}}{5676}\right) \\
& -\frac{1512 \ln (240)}{4489}-\frac{64368 \sqrt{1419} \arctan \left(\frac{17 \sqrt{1419}}{473}\right)}{2123297},
\end{aligned}
$$

which generates the following approximate distance modulus,

$$
(m-M)_{22}=5 \frac{\ln \left(5 / 3 R_{0}(1+z) I_{G 22}(z)\right)}{\ln (10)}+25 \text {. }
$$

The percent error between the approximate distance modulus as given by Equation (58) and the exact distance modulus as given by Equation (52) is $\approx 0.03 \%$ when $z=4$ and $H_{0}=69.1$.

\subsection{Simple GR Cosmology}

In the framework of GR, the received flux, $f$, is

$$
f=\frac{L}{4 \pi D_{L}^{2}}
$$

where $D_{L}$ is the luminosity distance, which depends on the cosmological model adopted, see Equation (7.21) in [40] or Equation (5.235) in [41].

The distance modulus in the simple GR cosmology is

$$
m-M=43.17-\frac{1}{\ln (10)} \ln \left(\frac{H_{0}}{70}\right)+5 \frac{\ln (z)}{\ln (10)}+1.086\left(1-q_{0}\right) z,
$$

see Equation (7.52) in [40]. There are two free parameters in the simple GR cosmology: $H_{0}$ and $q_{0}$.

\subsection{Flat Expanding Universe}

This model is based on the standard definition of luminosity in the flat expanding universe. The luminosity distance, $r_{L}^{\prime}$, is

$$
r_{L}^{\prime}=\frac{c}{H_{0}} z
$$

and the distance modulus is

$$
m-M=-5 \log _{10}+5 \log _{10} r_{L}^{\prime}+2.5 \log (1+z),
$$

see formulae (13) and (14) in [42]. There is one free parameter in the flat ex- 
panding model, $H_{0}$.

\subsection{The Milne Universe in SR}

In the Milne model, which is developed in the framework of SR, the luminosity distance, after [43] [44] [45], is

$$
D_{L}=\frac{c\left(z+\frac{1}{2} z^{2}\right)}{H_{0}},
$$

and the distance modulus for the Milne model is

$$
m-M=25+5 \frac{1}{\ln (10)} \ln \left(\frac{c\left(z+\frac{1}{2} z^{2}\right)}{H_{0}}\right) .
$$

There is one free parameter in the Milne model: $H_{0}$.

\subsection{Plasma Cosmology}

In a Euclidean static framework from among many possible absorption mechanisms, we have selected a plasma effect which produces the following relation for the distance $d$,

$$
d=\frac{c}{H_{0}} \ln (1+z),
$$

where the distance expressed in lower case underline the difference with the relativistic case, see Equation (50) in [46].

In the presence of plasma absorption, the observed flux is

$$
f=\frac{L \cdot \exp \left(-b d-H_{0} d-2 H_{0} d\right)}{4 \pi d^{2}},
$$

where the factor $\exp (-b d)$ is due to galactic and host galactic extinctions, $-H_{0} d$ is the reduction due to the plasma in the IGM and $-2 H_{0} d$ is the reduction due to the Compton scattering, see the formula before Equation (51) in [46]. The resulting distance modulus in the plasma mechanism is

$$
m-M=5 \frac{\ln (\ln (z+1))}{\ln (10)}+\frac{15}{2} \frac{\ln (z+1)}{\ln (10)}+5 \frac{1}{\ln (10)} \ln \left(\frac{c}{H_{0}}\right)+25+1.086 b,
$$

see Equation (7) in [47]. There is one free parameter in the plasma cosmology: $H_{0}$ when $b=0$. A detailed analysis of this and other physical mechanisms which produce the observed redshift can be found in [48].

\subsection{Modified Tired Light}

In a Euclidean static universe, the concept of modified tired light (MTL) was introduced in Section 2.2 of [49]. The distance in the MTL is

$$
d=\frac{c}{H_{0}} \ln (1+z),
$$


where the distance expressed in lower case underline the difference with the relativistic case. The distance modulus in MTL is

$$
m-M=\frac{5}{2} \frac{\beta \ln (z+1)}{\ln (10)}+5 \frac{1}{\ln (10)} \ln \left(\frac{\ln (z+1) c}{H_{0}}\right)+25,
$$

where $\beta$ is a parameter lying between 1 and 3 which allows matching theory with observations. There are two free parameters in MTL: $H_{0}$ and $\beta$.

\section{Astrophysical Results}

We first review the statistics involved and then we process the $12 \times 4$ cosmological cases.

\subsection{The Adopted Statistics}

In the case of the distance modulus, the merit function $\chi^{2}$ is

$$
\chi^{2}=\sum_{i=1}^{N}\left[\frac{(m-M)_{i}-(m-M)\left(z_{i}\right)_{t h}}{\sigma_{i}}\right]^{2},
$$

where $N$ is the number of SNs, $(m-M)_{i}$ is the observed distance modulus evaluated at a redshift of $z_{i}, \sigma_{i}$ is the error in the observed distance modulus evaluated at $z_{i}$, and $(m-M)\left(z_{i}\right)_{t h}$ is the theoretical distance modulus evaluated at $z_{i}$, see formula (15.5.5) in [50]. The reduced merit function $\chi_{\text {red }}^{2}$ is:

$$
\chi_{\text {red }}^{2}=\chi^{2} / N F,
$$

where $N F=N-k$ is the number of degrees of freedom, $N$ is the number of SNs, and $k$ is the number of free parameters. Another useful statistical parameter is the associated $Q$-value, which has to be understood as the maximum probability of obtaining a better fitting, see formula (15.2.12) in [50]:

$$
Q=1-\operatorname{GAMMQ}\left(\frac{N-k}{2}, \frac{\chi^{2}}{2}\right),
$$

where GAMMQ is a subroutine for the incomplete gamma function. The Akaike information criterion (AIC), see [51], is defined by

$$
\mathrm{AIC}=2 k-2 \ln (L),
$$

where $L$ is the likelihood function. We assume a Gaussian distribution for the errors; then the likelihood function can be derived from the $\chi^{2}$ statistic $L \propto \exp \left(-\frac{\chi^{2}}{2}\right)$ where $\chi^{2}$ has been computed by Equation (70), see [52] [53].

Now the AIC becomes

$$
\mathrm{AIC}=2 k+\chi^{2} .
$$

The goodness of the approximation in evaluating a physical variable $p$ is evaluated by the percentage error $\delta$,

$$
\delta=\frac{\left|p-p_{\text {approx }}\right|}{p} \times 100,
$$


where $p_{\text {approx }}$ is an approximation of $p$.

\subsection{The Numerical Techniques}

The parameters of the twelve cosmologies here analyzed are found minimizing the $\chi^{2}$ as given by Equation (70). We now report the adopted numerical techniques:

1) In absence of an analytical solution for the distance modulus we do $\mathrm{k}$ (the number of free parameters) nested numerical loops for the evaluation of the $\chi^{2}$. The parameters which minimize the $\chi^{2}$ are selected. This method allows to find, as an example, the parameters of the $\Lambda$ CDM and $\phi C D M$ cosmologies.

2) In presence of an analytical solution, an approximate Taylor series and a Padé approximant for the distance modulus we derive the parameters through the Levenberg-Marquardt method (subroutine MRQMIN in [50]) once an analytical expression for the derivatives of the distance modulus with respect to the unknown parameters is provided. In absence of a human expression for the derivatives, we implement the numerical derivative. This method was used to evaluate the parameters of the MTL, the simple GR, the plasma, the Milne, the Einstein-De Sitter, the flat, the wCDM and the Cardassian cosmologies.

The above techniques allow to derive the cosmological parameters with unprecedented accuracy, as an example, an error of $0.1 \mathrm{~km} \cdot \mathrm{s}^{-1} \cdot \mathrm{Mpc}^{-1}$ can be associated with the Hubble constant. The advantage to have approximate results, i.e. the Padé approximant for the distance modulus $(m-M)_{2,2}$ as given by Equation (11), is that we can evaluate in an analytical way the first derivative required by the Levenberg-Marquardt method and the numerical integration is not necessary.

\subsection{The Four Compilations}

In order to avoid the degeneracy in the Hubble constant-absolute magnitude plane we deal only with already calibrated distance modulus. The first astronomical test we perform is on the 580 SNs of the Union 2.1 compilation, see [5], which is available at

http://supernova.lbl.gov/Union/figures/SCPUnion2.1_mu_vs_z.txt: in this compilation a calibrated distance versus redshift is provided. The cosmological parameters are reported in Table 1 and Figure 3 reports the best fit in the $\Lambda$ CDM cosmology.

The second test we perform is on the joint light-curve analysis (JLA), which contains 740 SNs [6] with data available on CDS at

http://cdsweb.u-strasbg.fr/. The above compilation consists of SNe (type I-a) for which we have a heliocentric redshift, $Z$, apparent magnitude $m_{B}^{\star}$ in the B band, error in $m_{B}^{\star}, \sigma_{m_{B}^{\star}}$, parameter $X 1$, error in $X 1, \sigma_{X 1}$, parameter $C$, error in the parameter $C, \sigma_{C}$ and $\log _{10}\left(M_{\text {stellar }}\right)$. The observed distance modulus is defined by Equation (4) in [6],

$$
m-M=-C \beta+X 1 \alpha-M_{b}+m_{B}^{\star} .
$$


Table 1. Numerical values of $\chi^{2}, \chi_{\text {red }}^{2}, Q$ and the AIC of the Hubble diagram for the Union 2.1 compilation: $k$ stands for the number of parameters, $H_{0}$ is expressed in $\mathrm{km} \cdot \mathrm{s}^{-1} \cdot \mathrm{Mpc}^{-1} ; 580 \mathrm{SNs}$.

\begin{tabular}{|c|c|c|c|c|c|c|c|}
\hline cosmology & Equation & $k$ & parameters & $\chi^{2}$ & $\chi_{\text {red }}^{2}$ & $Q$ & AIC \\
\hline$\Lambda \mathrm{CDM}$ & (11) & 3 & $H_{0}=69.56 \pm 0.1 ; \Omega_{\mathrm{M}}=0.238 \pm 0.01 ; \Omega_{\Lambda}=0.661 \pm 0.01$ & 562.59 & 0.975 & 0.658 & 569.39 \\
\hline $\mathrm{wCDM}$ & (23) & 3 & $H_{0}=70.02 \pm 0.35 ; \quad \Omega_{\mathrm{M}}=0.277 \pm 0.025 ; \quad w=-1.003 \pm 0.05$ & 562.21 & 0.974 & 0.662 & 568.21 \\
\hline Cardassian & (31) & 3 & $H_{0}=70.15 \pm 0.38 ; \quad \Omega_{\mathrm{M}}=0.305 \pm 0.019 ; \quad n=-0.081 \pm 0.01$ & 562.35 & 0.974 & 0.661 & 568.35 \\
\hline flat & (39) & 2 & $H_{0}=69.77 \pm 0.33 ; \Omega_{\mathrm{M}}=0.295 \pm 0.008$ & 562.55 & 0.9732 & 0.66 & 566.55 \\
\hline$\phi \mathrm{CDM}$ & (46) & 4 & $\begin{aligned} H_{0}=70 \pm 0.1 ; \quad \Omega_{\mathrm{M} 0} & =0.28 \pm 0.02 ; \alpha=-0.08 \pm 0.2 ; \\
\beta & =0.05 \pm 0.02\end{aligned}$ & 562.23 & 0.976 & 0.65 & 570.23 \\
\hline Einstein--De Sitter & (51) & 1 & $H_{0}=63.17 \pm 0.2$ & 1171.39 & 2.02 & $2 \times 10^{-42}$ & 1173.39 \\
\hline EdesNa & (52) & 1 & $H_{0}=69.04 \pm 0.22$ & 569.46 & 0.98 & 0.603 & 571.46 \\
\hline simple GR & $(60)$ & 2 & $H_{0}=73.79 \pm 0.024, \quad q_{0}=-0.1$ & 689.34 & 1.194 & $9.5 \times 10^{-4}$ & 693.34 \\
\hline flat expanding model & (62) & 1 & $H_{0}=66.84 \pm 0.22$ & 653 & 1.12 & 0.017 & 655 \\
\hline Milne & (64) & 1 & $H_{0}=67.53 \pm 0.22$ & 603.37 & 1.04 & 0.23 & 605.37 \\
\hline plasma & $(67)$ & 1 & $H_{0}=74.2 \pm 0.24$ & 895.53 & 1.546 & $5.2 \times 10^{-16}$ & 6897.5 \\
\hline MTL & (69) & 2 & $\beta=2.37, \quad H_{0}=69.32 \pm 0.34$ & 567.96 & 0.982 & 0.609 & 571.9 \\
\hline
\end{tabular}

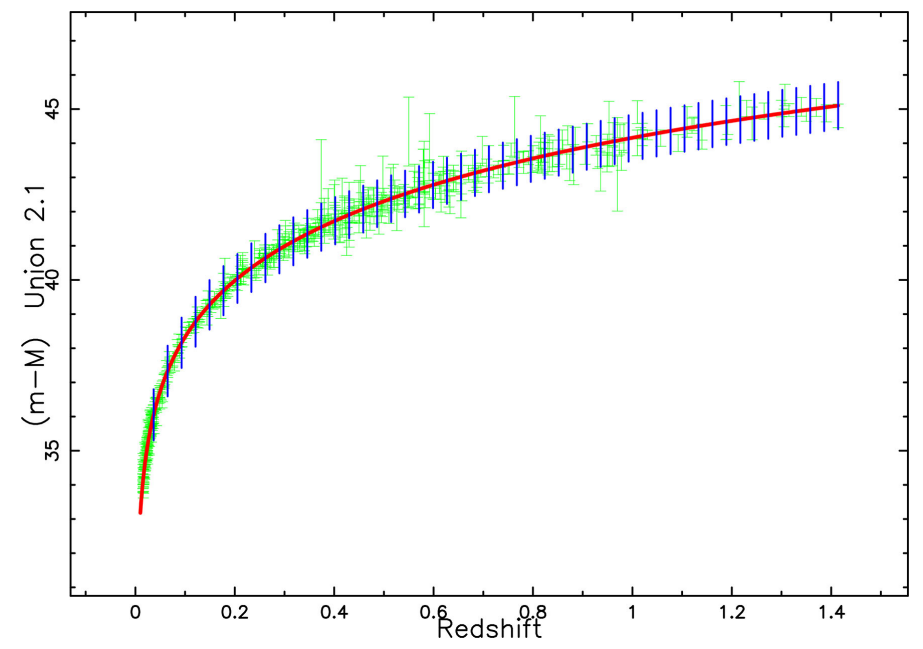

Figure 3. Hubble diagram for the Union 2.1 compilation, green points with error bar. The solid red line represents the best fit for the distance modulus in $\Lambda \mathrm{CDM}$ cosmology as represented by Equation (11). The theoretical uncertainties are represented through blue vertical lines by applying the law of errors of Gauss with the uncertainties and parameters as in the first line of Table 1.

The adopted parameters are $\alpha=0.141, \beta=3.101$ and

$$
M_{b}=\left\{\begin{array}{ll}
-19.05 & \text { if } M_{\text {stellar }}<10^{10} M_{\odot} \\
-19.12 & \text { if } M_{\text {stellar }} \geq 10^{10} M_{\odot}
\end{array},\right.
$$

where $M_{\odot}$ is the mass of the sun, see line 1 in Table 10 of [6]. The uncertainty in the observed distance modulus, $\sigma_{m-M}$, is found by implementing the error propagation equation (often called the law of errors of Gauss) when the covariant terms are neglected, see Equation (3.14) in [54], 


$$
\sigma_{m-M}=\sqrt{\alpha^{2} \sigma_{X 1}^{2}+\beta^{2} \sigma_{C}^{2}+\sigma_{m_{B}^{ \pm}}^{2}} .
$$

The cosmological parameters with the JLA compilation are reported in see Table 2 and Figure 4 reports the best fit in the MTL cosmology.

The third test is performed on the Union 2.1 compilation (580 SNs) + the distance modulus for 59 calibrated high-redshift GRBs, the so called "Hymnium" sample of GRBs, which allows to calibrate the distance modulus in the high redshift up to $z \approx 8$ [55], see Table 3 and Figure 5 for the best fit in the Cardassian cosmology.

Table 2. Numerical values of $\chi^{2}, \chi_{\text {red }}^{2}, Q$ and the AIC of the Hubble diagram for the JLA compilation, $k$ stands for the number of parameters, $H_{0}$ is expressed in $\mathrm{km} \cdot \mathrm{s}^{-1} \cdot \mathrm{Mpc}^{-1} ; 740 \mathrm{SNs}$.

\begin{tabular}{cccccccc}
\hline cosmology & Equation & $k$ & parameters & $\chi^{2}$ & $\chi_{\text {red }}^{2}$ & $Q$ & AIC \\
\hline$\Lambda$ CDM & $(11)$ & 3 & $H_{0}=70.71 \pm 0.1 ; \Omega_{\mathrm{M}}=0.238 \pm 0.01 ; \Omega_{\Lambda}=0.621 \pm 0.01$ & 626.53 & 0.85 & 0.998 & 632.53 \\
wCDM & $(23)$ & 3 & $H_{0}=69.38 \pm 0.31 ; \Omega_{\mathrm{M}}=0.2 \pm 0.016 ; w=-0.8 \pm 0.031$ & 626.01 & 0.849 & 0.998 & 632.01 \\
Cardassian & $(31)$ & 3 & $H_{0}=70.03 \pm 0.44 ; \Omega_{\mathrm{M}}=0.3 \pm 0.019 ; n=-0.055 \pm 0.004$ & 628.73 & 0.853 & 0.998 & 634.73 \\
flat & $(39)$ & 2 & $H_{0}=69.65 \pm 0.23 ; \Omega_{\mathrm{M}}=0.3 \pm 0.003$ & 627.91 & 0.85 & 0.998 & 631.91 \\
CDDM & $(46)$ & 4 & $H_{0}=69.6 \pm 0.1 ; \Omega_{\mathrm{M} 0}=0.24 \pm 0.02 ; \alpha=0.31 \pm 0.2 ; \beta=0.03 \pm 0.02$ & 626.52 & 0.851 & 0.998 & 634.52 \\
Einstein--De Sitter & $(51)$ & 1 & $H_{0}=62.57 \pm 0.17$ & 1307.75 & 1.76 & $3.27 \times 10^{-34}$ & 1309.75 \\
EdesNa & $(52)$ & 1 & $H_{0}=68.91 \pm 0.19$ & 630.46 & 0.853 & 0.998 & 632.46 \\
simple GR & $(60)$ & 2 & $H_{0}=73.79 \pm 0.023, q_{0}=-0.14$ & 749.14 & 1.016 & 0.369 & 755.14 \\
flat expanding model & $(62)$ & 1 & $H_{0}=66.49 \pm 0.18$ & 717.3 & 0.97 & 0.709 & 719.3 \\
Milne & $(64)$ & 1 & $H_{0}=67.19 \pm 0.18$ & 656.11 & 0.887 & 0.986 & 658.11 \\
plasma & $(67)$ & 1 & $H_{0}=74.45 \pm 0.2$ & 1017.79 & 1.377 & $3.59 \times 10^{-11}$ & 1019.79 \\
MTL & $(69)$ & 2 & $\beta=2.36, H_{0}=69.096 \pm 0.32$ & 626.27 & 0.848 & 0.998 & 630.27 \\
\hline
\end{tabular}

Table 3. Numerical values of $\chi^{2}, \chi_{\text {red }}^{2}, Q$ and the AIC of the Hubble diagram for the Union 2.1 compilation + the "Hymnium" GRB sample, $k$ stands for the number of parameters, $H_{0}$ is expressed in $\mathrm{km} \cdot \mathrm{s}^{-1} \cdot \mathrm{Mpc}^{-1} ; 580 \mathrm{SNs}+59 \mathrm{GRBs}$.

\begin{tabular}{|c|c|c|c|c|c|c|c|}
\hline cosmology & Equation & $k$ & parameters & $\chi^{2}$ & $\chi_{\text {red }}^{2}$ & $Q$ & AIC \\
\hline$\Lambda \mathrm{CDM}$ & $(11)$ & $H_{0}=67$ & $7.8 \pm 0.2 ; \quad \Omega_{\mathrm{M}}=0.259 \pm 0.02 ; \Omega_{\Lambda}=0.691 \pm 0.02$ & 586.04 & 0.921 & 0.922 & 592.04 \\
\hline $\mathrm{wCDM}$ & $(23)$ & $H_{0}=69.3$ & $34 \pm 0.32 ; \quad \Omega_{\mathrm{M}}=0.2 \pm 0.016 ; \quad w=-0.626 \pm 0.015$ & 592.1 & 0.93 & 0.892 & 598.1 \\
\hline Cardassian & $(31)$ & $H_{0}=70.1$ & $1 \pm 0.42 ; \Omega_{\mathrm{M}}=0.299 \pm 0.019 ; \quad n=-0.063 \pm 0.009$ & 585.43 & 0.92 & 0.924 & 591.43 \\
\hline flat & $(39)$ & 2 & $H_{0}=69.82 \pm 0.24 ; \Omega_{\mathrm{M}}=0.295 \pm 0.003$ & 585.74 & 0.919 & 0.927 & 589.74 \\
\hline$\phi \mathrm{CDM}$ & $(46)$ & $H_{0}=70 \pm 0.1$ & $; \quad \Omega_{\mathrm{M} 0}=0.28 \pm 0.02 ; \alpha=-0.07 \pm 0.2 ; \beta=0.05 \pm 0.02$ & 585.41 & 0.922 & 0.92 & 593.41 \\
\hline Einstein--De Sitter & $(51)$ & 1 & $H_{0}=63.14 \pm 0.2$ & 1205.2 & 1.88 & $3.58 \times 10^{-37}$ & 1205.21 \\
\hline Edes Na & $(52)$ & 1 & $H_{0}=69.05 \pm 0.22$ & 592.79 & 0.929 & 0.899 & 594.79 \\
\hline simple GR & $(60)$ & 2 & $H_{0}=73.79 \pm 0.023, \quad q_{0}=-0.01$ & 809.5 & 1.27 & $3.85 \times 10^{-6}$ & 813.5 \\
\hline flat expanding model & $(62)$ & 1 & $H_{0}=66.851 \pm 0.22$ & 676.36 & 1.06 & 0.141 & 678.36 \\
\hline Milne & $(64)$ & 1 & $H_{0}=67.55 \pm 0.22$ & 634.27 & 0.994 & 0.534 & 636.27 \\
\hline plasma & $(67)$ & 1 & $H_{0}=74.25 \pm 0.24$ & 951.16 & 1.49 & $9.39 \times 10^{-14}$ & 953.16 \\
\hline MTL & $(69)$ & 2 & $\beta=2.35, \quad H_{0}=69.23 \pm 0.34$ & 594.69 & 0.933 & 0.883 & 598.69 \\
\hline
\end{tabular}




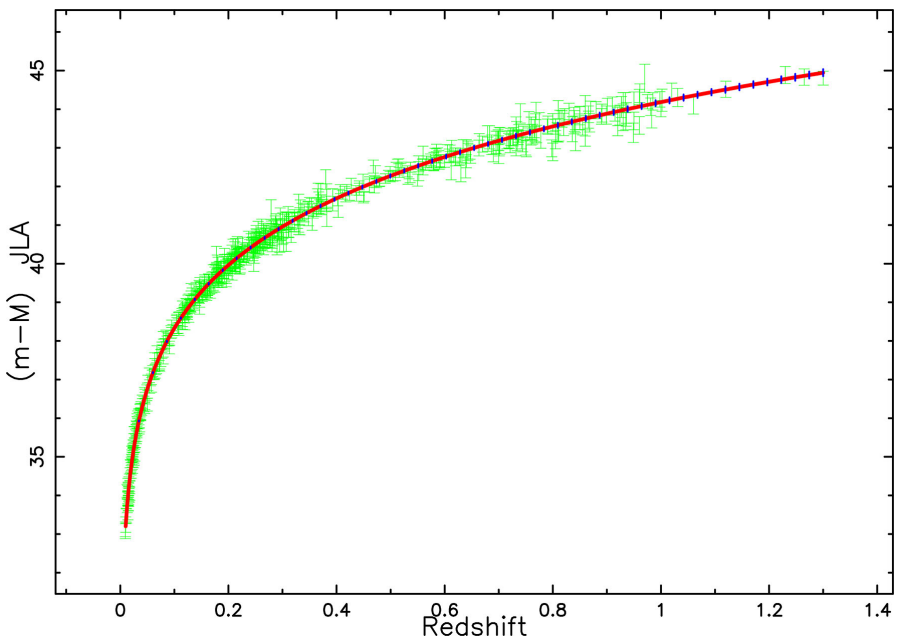

Figure 4. Hubble diagram for the JLA compilation, green points with error bar. The solid red line represents the best fit for the distance modulus in MTL cosmology as represented by Equation (69). The theoretical uncertainties are represented through blue vertical lines.

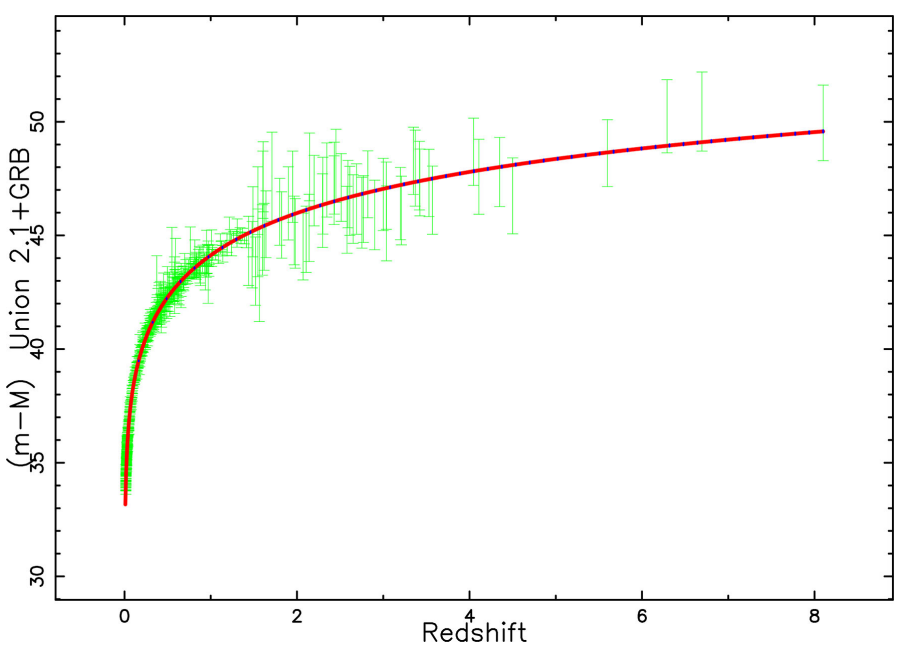

Figure 5. Hubble diagram for the Union 2.1 compilation + the "Hymnium" GRB sample, green points with error bar. The solid red line represents the best fit for the distance modulus in Cardassian cosmology as represented by Equation (31). The theoretical uncertainties are represented through blue vertical lines.

The fourth test is performed on the Pantheon sample of 1048 SN Ia [7] [8] with calibrated data available at https://archive.stsci.edu/prepds/ps1cosmo/jones_datatable.html, see Table 4 and Figure 6 for the best fit in the flat cosmology.

In order to see how $\chi^{2}$ varies around the minimum for the Pantheon sample in the case of the $\Lambda \mathrm{CDM}$ cosmology, Figure 7 presents a $2 \mathrm{D}$ colour map for the values of $\chi^{2}$ for the Pantheon sample when $H_{0}$ and $\Omega_{\mathrm{M}}$ are allowed to vary around the numerical values which fix the minimum.

Figure 8 presents the map for $\chi^{2}$, for wCDM and for the Pantheon sample when $H_{0}$ is fixed and $\Omega_{\mathrm{M}}$ and $w$ are allowed to vary. 


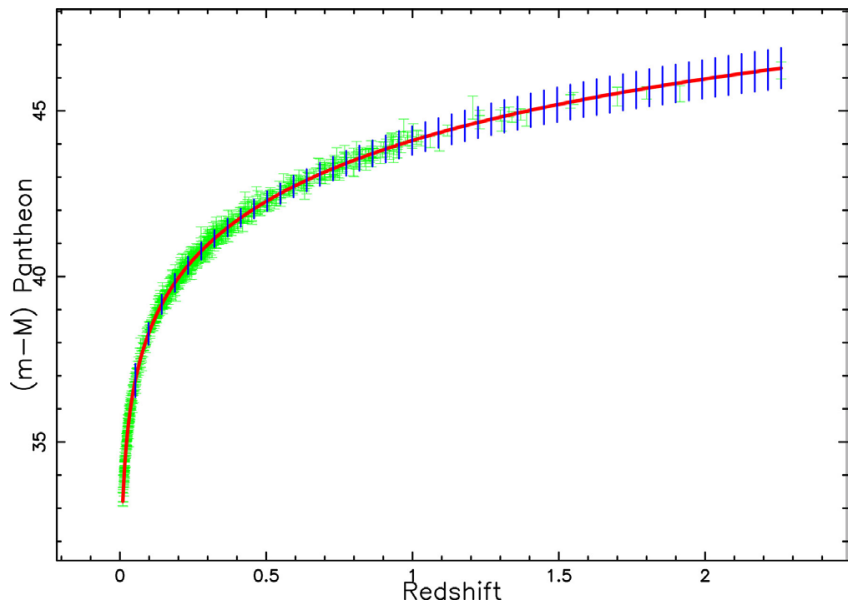

Figure 6. Hubble diagram for the Pantheon sample, green points with error bar. The solid red line represents the best fit for the distance modulus in flat cosmology as represented by Equation (39). The theoretical uncertainties are represented through blue vertical lines.

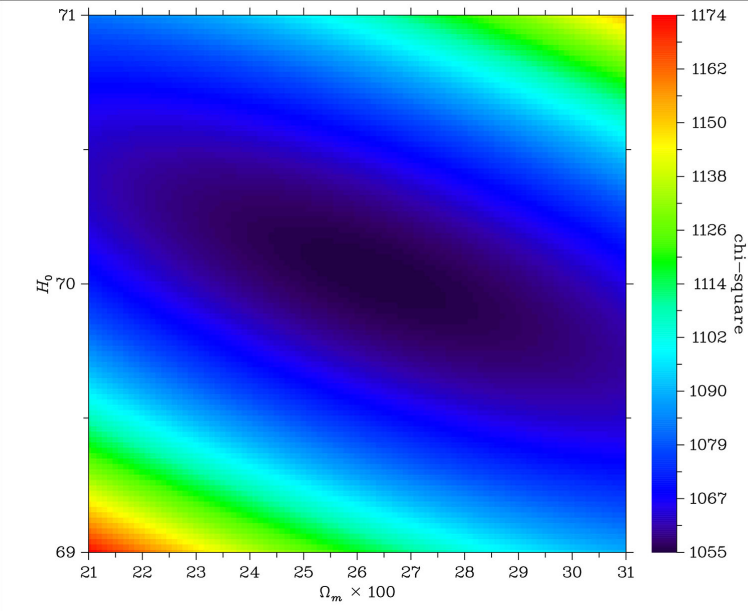

Figure 7. Color contour plot for $\chi^{2}$ of the Hubble diagram for the Pantheon sample in $\Lambda \mathrm{CDM}$ cosmology when $H_{0}$ and $\Omega_{\mathrm{M}}$ are variables and $\Omega_{\Lambda}=0.626$.

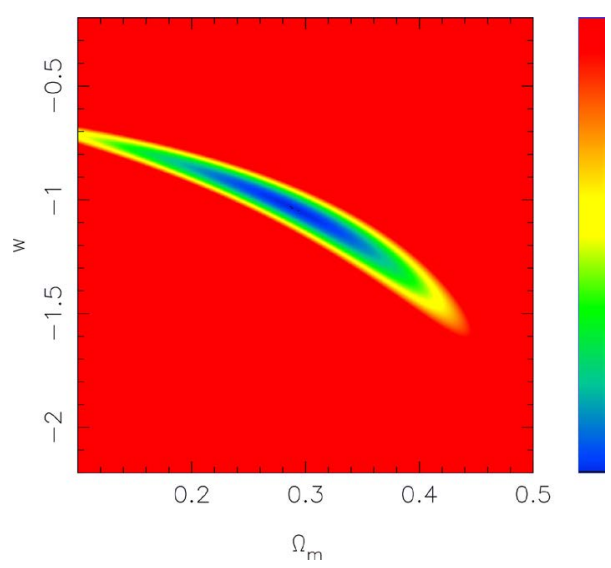

Figure 8. Map of $\chi^{2}$ for the wCDM cosmology when $H_{0}=69.8$. 
Table 4. Numerical values of $\chi^{2}, \chi_{\text {red }}^{2}, Q$ and the AIC of the Hubble diagram for the Pantheon sample, $k$ stands for the number of parameters, $H_{0}$ is expressed in $\mathrm{km} \cdot \mathrm{s}^{-1} \cdot \mathrm{Mpc}^{-1} ; 1048$ SN Ia.

\begin{tabular}{|c|c|c|c|c|c|c|c|}
\hline cosmology & Equation & $k$ & parameters & $\chi^{2}$ & $\chi_{\text {red }}^{2}$ & $Q$ & AIC \\
\hline$\Lambda \mathrm{CDM}$ & $(11)$ & 3 & $H_{0}=68.209 \pm 0.2 ; \Omega_{\mathrm{M}}=0.278 \pm 0.02 ; \Omega_{\Lambda}=0.651 \pm 0.02$ & 1054.71 & 1.01 & 0.41 & 1060.71 \\
\hline wCDM & (23) & 3 & $H_{0}=69.8 \pm 0.27 ; \Omega_{\mathrm{M}}=0.3 \pm 0.016 ; \quad w=-0.989 \pm 0.03$ & 1053.67 & 1 & 0.419 & 1059.67 \\
\hline Cardassian & $(31)$ & 3 & $H_{0}=70.01 \pm 0.31 ; \Omega_{\mathrm{M}}=0.329 \pm 0.014 ; n=-0.091 \pm 0.005$ & 1054.49 & 1 & 0.412 & 1060.49 \\
\hline flat & (39) & 2 & $H_{0}=69.94 \pm 0.171 ; \Omega_{\mathrm{M}}=0.296 \pm 0.002$ & 1053.53 & 1 & 0.429 & 1057.53 \\
\hline$\phi \mathrm{CDM}$ & (46) & 4 & $\begin{array}{c}H_{0}=69.7 \pm 0.1 ; \quad \Omega_{\mathrm{M} 0}=0.28 \pm 0.02 ; \alpha=0.12 \pm 0.2 ; \\
\beta=0.05 \pm 0.02\end{array}$ & 1053.84 & 1 & 0.4 & 1061.84 \\
\hline Einstein--De Sitter & (51) & 1 & $H_{0}=62.71 \pm 0.2$ & 2387.62 & 2.28 & 0 & 2389.62 \\
\hline Edes Na & (52) & 1 & $H_{0}=69.1 \pm 0.13$ & 1059.84 & 1.01 & 0.384 & 1061.8 \\
\hline simple GR & $(60)$ & 2 & $H_{0}=73.79 \pm 0.015, \quad q_{0}=-0.063$ & 1476.59 & 1.411 & $2.67 \times 10^{-17}$ & 1480.59 \\
\hline flat expanding model & (62) & 1 & $H_{0}=66.67 \pm 0.12$ & 1219 & 1.16 & $1.6 \times 10^{-4}$ & 1221 \\
\hline Milne & $(64)$ & 1 & $H_{0}=67.37 \pm 0.12$ & 1132.6 & 1.08 & 0.033 & 1134.6 \\
\hline plasma & (67) & 1 & $H_{0}=74.7 \pm 0.14$ & 2017.3 & 1.92 & 0 & 2019.3 \\
\hline MTL & (69) & 2 & $\beta=2.31, \quad H_{0}=68.95 \pm 0.222$ & 1069.7 & 1.022 & 0.298 & 1073.7 \\
\hline
\end{tabular}

\subsection{Angular-Diameter Distance}

In the relativistic models the angular diameter distance, $D_{\mathrm{A}} \quad[56]$, is

$$
D_{\mathrm{A}}=\frac{D_{\mathrm{L}}}{(1+z)^{2}} \text {. }
$$

We now introduce the minimax approximation. Let $f(x)$ be a real function defined in the interval $[a, b]$. The best rational approximation of degree $(k, l)$ evaluates the coefficients of the ratio of two polynomials of degree $k$ and $l$, respectively, which minimizes the maximum difference of:

$$
\max \left|f(x)-\frac{p_{0}+p_{1} x+\cdots+p_{k} x^{k}}{q_{0}+q_{1} x+\cdots+q_{\ell} x^{\ell}}\right|,
$$

on the interval $[a, b]$. The quality of the fit is given by the maximum error over the considered range. The coefficients are evaluated through the Remez algorithm, see [57] [58]. The minimax approximation for the angular distance in the interval $0<z<8$ with data as in Table 3 for $\Lambda$ CDM cosmology when $k=2$ and $p=2$ is:

$$
\begin{gathered}
D_{A, 2,2}=\frac{-0.08126207+(296.9974312+2.715947207 z) z}{0.0672056121+(0.0810298760+0.02498056665 z) z} \mathrm{Mpc} \\
\text { maximum error }=0.6911273 \mathrm{Mpc},
\end{gathered}
$$

for wCDM cosmology when $k=3$ and $p=2$ is:

$$
\begin{gathered}
D_{A, 3,2}=\frac{0.034977336+(287.18685+(1.1871126+0.0002567152 z) z) z}{0.0665238+(0.09134443+0.023282807 z) z} \mathrm{Mpc}(82) \\
\text { maximum error }=0.07 \mathrm{Mpc},
\end{gathered}
$$


for Cardassian cosmology when $k=2$ and $p=2$ is:

$$
\begin{gathered}
D_{A, 2,2}=\frac{-0.11928613+(273.3160492+2.420885784 z) z}{0.0638700712+(0.0750594027+0.02611741351 z) z} \mathrm{Mpc} \\
\text { maximum error }=0.8346776 \mathrm{Mpc},
\end{gathered}
$$

for flat cosmology when $k=2$ and $p=2$ is:

$$
\begin{gathered}
D_{A, 2,2}=\frac{-0.03653022+(274.6370918+2.192330157 z) z}{0.0641307653+(0.0767316787+0.02582682170 z) z} \mathrm{Mpc} \\
\text { maximum error }=0.629004 \mathrm{Mpc},
\end{gathered}
$$

and for $\phi \mathrm{CDM}$ cosmology when $k=2$ and $p=2$ is:

$$
\begin{gathered}
D_{A, 2,2}=\frac{-0.01852238+(278.5646306+2.230340777 z) z}{0.0652823706+(0.0768568011+0.02575830541 z) z} \\
\text { maximum error }=0.6261293 \mathrm{Mpc} .
\end{gathered}
$$

In MTL there is no difference between the distance $d$, see Equation (68), and the angular distance. We report the numerical value of $d$ in the interval $0<z<8$ with data as in Table 3,

$$
d=4330.383620 \ln (z+1) \text { Mpc. }
$$

A promising field of investigation in applied cosmology is the maximum of the angular distance as function of the redshift [59] [60], $z_{\max }$, which is finite in relativistic cosmologies and infinite in the Milne, plasma and MTL cosmologies, see Figure 9.

The numerical value of $z_{\max }$ is reported in Table 5, as a reference $z_{\max }=1.594$ for flat Planck $\Lambda$ CDM cosmology [61].

Another example is given by the ring associated with the galaxy SDP.81, see [62], which is generally explained by the gravitational lens. In this framework we have a foreground galaxy at $z=0.2999$ and a background galaxy at $z=0.3042$. This ring has been studied with the Atacama Large Millimeter/sub-millimeter Array (ALMA) by [63]-[68]. The system SDP.81 has been analysed by ALMA and presents 14 molecular clumps along the two main lensed arcs: the averaged radius in arcsec is $R_{\text {ave }}=1.54 \operatorname{arcsec}$ [69].

Table 5. Numerical values of $z_{\max }$ and radius of Einstein ring in kpc when $R_{\text {ave }}=1.54 \mathrm{arcsec}$.

\begin{tabular}{ccc}
\hline cosmology & $z_{\max }$ & radius $(\mathrm{kpc})$ \\
\hline$\Lambda \mathrm{CDM}$ & 1.691 & 13.333 \\
$\mathrm{wCDM}$ & 1.716 & 11.797 \\
Cardassian & 1.607 & 11.938 \\
flat & 1.615 & 11.907 \\
$\phi \mathrm{CDM}$ & 1.632 & 12.05 \\
$\mathrm{MTL}$ & $\infty$ & 45.15 \\
\hline
\end{tabular}


Table 6. The first, second, third and fourth best fitting models for the four compilations.

\begin{tabular}{ccccc}
\hline Compilation & first model & second model & third model & fourth model \\
\hline Union 2.1 & $\begin{array}{c}\text { wCDM } \\
\text { Hypergeometric } \\
\text { JLA }\end{array}$ & Cardassian & $\phi$ CDM & flat \\
Hypergeometric & MTL & $\phi$ CDM & $\Lambda$ CDM \\
Union 2.1 + GRBs & $\begin{array}{c}\Lambda \text { CDM } \\
\text { wCDM } \\
\text { Pantheon }\end{array}$ & $\phi$ CDM & Cardassian & flat \\
& Hypergeometric & Cardassian & flat & $\phi \mathrm{CDM}$ \\
\hline
\end{tabular}

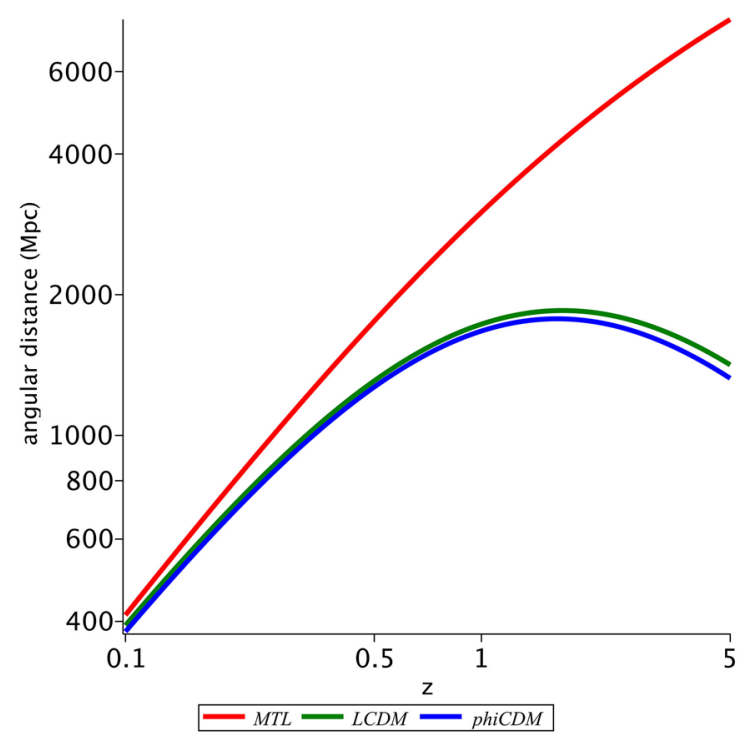

Figure 9. Angular distance in MTL (red), $\Lambda \mathrm{CDM}$ (green) and $\phi \mathrm{CDM}$ (blue) cosmologies with data as in Table 3.

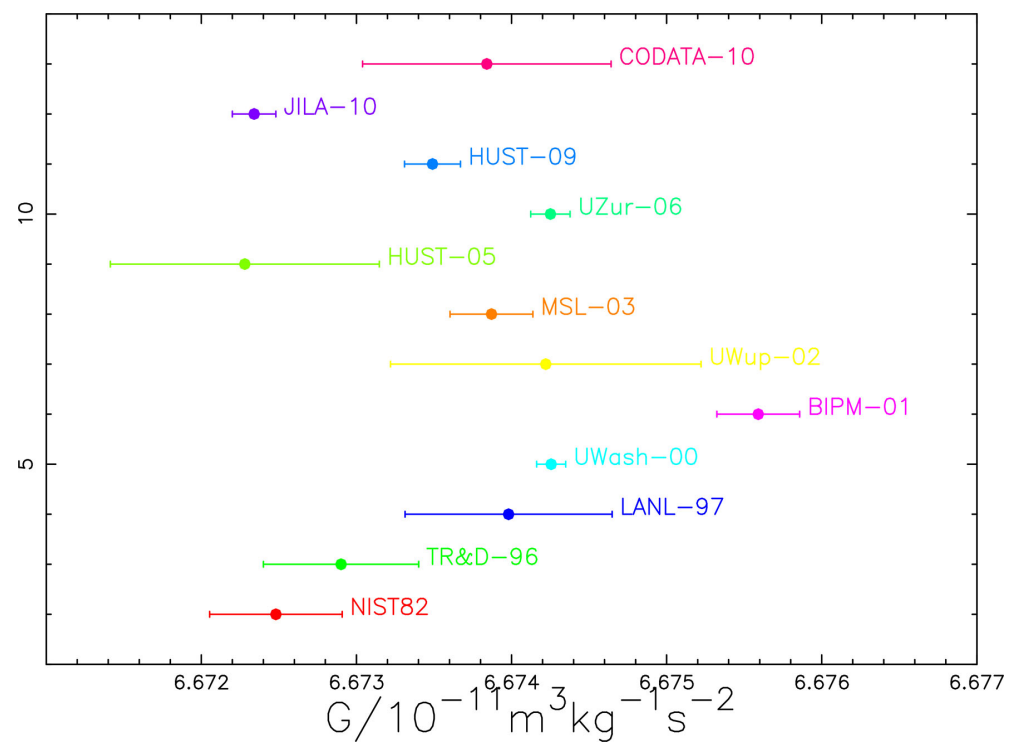

Figure 10. Values of the Newtonian constant of gravitation $G$ as given by Table XXIV in [70]. 


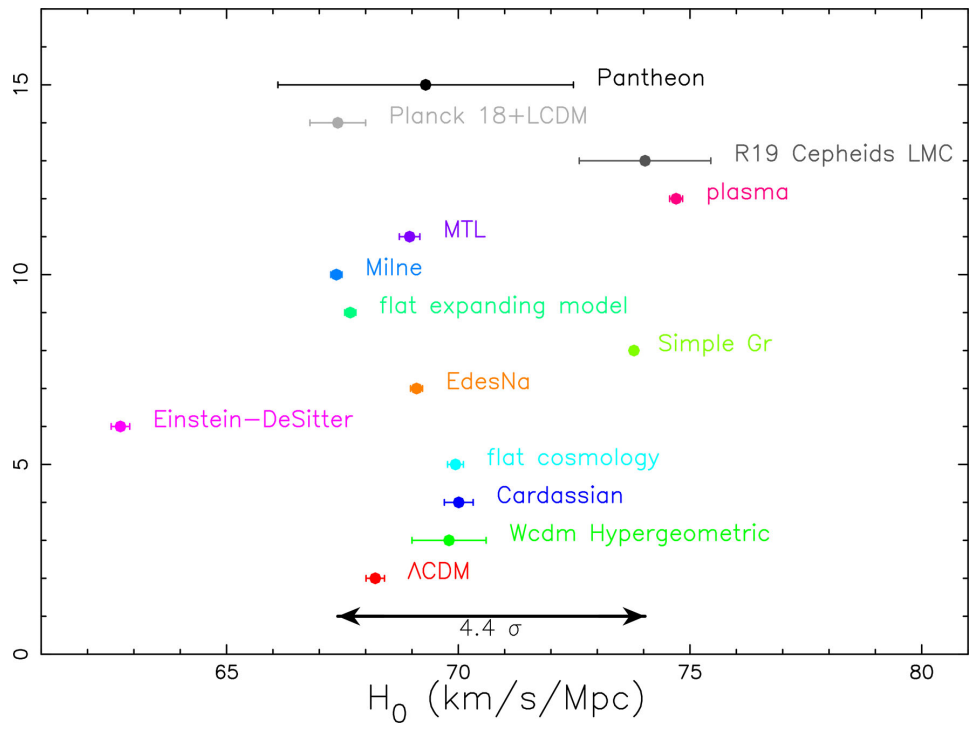

Figure 11. The present tension on $H_{0}$ (black line with two arrows) and our results in the case of the Pantheon sample with the connected averaged value which is marked as "Pantheon"; parameters as in Table 4.

\section{Conclusions}

Cosmological models: We list according to increasing order of the values of the merit function, $\chi^{2}$, the first, second, third, and fourth cosmological models, see Table 6.

The Einstein-De Sitter, simple GR, and plasma models produce the highest values in the $\chi^{2}$ and are here considered only for historical reasons.

Physics versus Astronomy: The value of the Newtonian gravitational constant, denoted by $G$, is derived applying the weighted mean, but the uncertainties were multiplied by a factor of 14, of 11 values available in Table XXIV in [70], see Figure 10.

By analogy, we average the values of $H_{0}$ for the Pantheon sample and we report as error for $H_{0}$ the standard deviation,

$$
\overline{H_{0}}=(69.29 \pm 3.18) \mathrm{km} \cdot \mathrm{s}^{-1} \cdot \mathrm{Mpc}^{-1} \quad \text { Pantheon sample, }
$$

see Figure 11.

\section{Acknowledgements}

The author is grateful to David Jones for information useful for downloading the data of the Pantheon sample.

\section{Conflicts of Interest}

The author declares no conflicts of interest regarding the publication of this paper.

\section{References}

[1] Planck Collaboration (2018) Planck 2018 Results. VI. Cosmological Parameters. ar- 
Xiv:1807.06209. (Preprint)

[2] Riess, A.G., Casertano S, Yuan W, Macri, L.M. and Scolnic, D. (2019) Large Magellanic Cloud Cepheid Standards Provide a 1\% Foundation for the Determination of the Hubble Constant and Stronger Evidence for Physics beyond $\Lambda$ CDM. The Astrophysical Journal, 876, Article No. 85. (Preprint) https://doi.org/10.3847/1538-4357/ab1422

[3] Di Valentino, E., Anchordoqui, L.A., Akarsu, O., Ali-Haimoud, Y., Amendola, L., Arendse, N., et al. (2020) Cosmology Intertwined II: The Hubble Constant Tension. arXiv:2008.11284. (Preprint)

[4] Riess, A.G., Filippenko, A.V., Challis, P. and Clocchiatti, A. (1998) Observational Evidence from Supernovae for an Accelerating Universe and a Cosmological Constant. The Astronomical Journal, 116, Article No. 1009. (Preprint) https://doi.org/10.1086/300499

[5] Suzuki, N., Rubin, D., Lidman, C., Aldering, G., Amanullah, R., Barbary, K. and Barrientos, L.F. (2012) The Hubble Space Telescope Cluster Supernova Survey. V. Improving the Dark-Energy Constraints above $\mathrm{Z}$ Greater than 1 and Building an Early-Type-Hosted Supernova Sample. The Astrophysical Journal, 746, Article No. 85. https://doi.org/10.1088/0004-637X/746/1/85

[6] Betoule, M., Kessler, R., Guy, J., Mosher, J., Hardin, D., Biswas, R., et al. (2014) Improved Cosmological Constraints from a Joint Analysis of the SDSS-II and SNLS Supernova Samples. Astronomy and Astrophysics, 568, Article No. A22. https://doi.org/10.1051/0004-6361/201423413

[7] Jones, D.O., Scolnic, D.M., Riess, A.G., Rest, A., Kirshner, R.P., Berger, E., et al. (2018) Measuring Dark Energy Properties with Photometrically Classified PanSTARRS Supernovae. II. Cosmological Parameters. The Astrophysical Journal, 857, Article No. 51. (Preprint) https://doi.org/10.3847/1538-4357/aab6b1

[8] Scolnic, D.M., Jones, D.O., Rest, A., Pan, Y.C., Chornock, R., Foley, R.J., et al. (2018) The Complete Light-curve Sample of Spectroscopically Confirmed SNe Ia from Pan-STARRS1 and Cosmological Constraints from the Combined Pantheon Sample. The Astrophysical Journal, 859, Article No. 101. (Preprint) https://doi.org/10.3847/1538-4357/aab9bb

[9] Oliveira, F.J. (2016) Cosmic Time Transformations in Cosmological Relativity Journal of High Energy Physics, Gravitation and Cosmology, 2, 253-279. https://doi.org/10.4236/jhepgc.2016.22022

[10] Gupta, R. (2018) SNe Ia Redshift in a Nonadiabatic Universe. Universe, 4, Article No. 104. (Preprint) https://doi.org/10.3390/universe4100104

[11] Amarzguioui, M., Elgarøy, Ø., Mota, D.F. and Multamäki, T. (2006) Cosmological Constraints on $\mathrm{f}(\mathrm{R})$ Gravity Theories within the Palatini Approach. $A \& A, 454$, 707-714. (Preprint) https://doi.org/10.1051/0004-6361:20064994

[12] Odintsov, S.D., Gómez, D.S.C. and Sharov, G.S. (2019) Testing Logarithmic Corrections to $\mathrm{R}^{2}$-Exponential Gravity by Observational Data. Physical Review D, 99, Article ID: 024003. https://doi.org/10.1103/PhysRevD.99.024003

[13] Corda, C. (2009) Interferometric Detection of Gravitational Waves: The Definitive Test for General Relativity International Journal of Modern Physics D, 18, 2275-2282. (Preprint) https://doi.org/10.1142/S0218271809015904

[14] Lin, H.N., Li, X. and Tang, L. (2019) Non-Parametric Reconstruction of Dark Energy and Cosmic Expansion from the Pantheon Compilation of Type Ia Supernovae Chinese Physics C, 43, Article ID: 075101. (Preprint) https://doi.org/10.1088/1674-1137/43/7/075101 
[15] Kazım Camlbel, A., Semiz, I. and Akif Feyizoğlu, M. (2020) Pantheon Update on a Model-Independent Analysis of Cosmological Supernova Data. Classical and Quantum Gravity, 37, Article ID: 235001. https://doi.org/10.1088/1361-6382/abba48

[16] Hogg, D.W. (1999) Distance Measures in Cosmology. arXiv:astro-ph/9905116. (Preprint)

[17] Peebles, P.J.E. (1993) Principles of Physical Cosmology. Princeton University Press, Princeton.

[18] Zaninetti, L. (2016) Pade Approximant and Minimax Rational Approximation in Standard Cosmology Galaxies, 4, Article No. 4. https://doi.org/10.3390/galaxies4010004

[19] Turner, M.S. and White, M. (1997) CDM Models with a Smooth Component. Physical Review D, 56, Article No. R4439. (Preprint) https://doi.org/10.1103/PhysRevD.56.R4439

[20] Tripathi, A., Sangwan, A. and Jassal, H.K. (2017) Dark Energy Equation of State Parameter and Its Evolution at Low Redshift. Journal of Cosmology and Astroparticle Physics, 6, Article No. 012. (Preprint) https://doi.org/10.1088/1475-7516/2017/06/012

[21] Wei, J.J., Ma, Q.B. and Wu, X.F. (2015) Utilizing the Updated Gamma-Ray Bursts and Type Ia Supernovae to Constrain the Cardassian Expansion Model and Dark Energy. Advances in Astronomy, 2015, Article ID: 576093. (Preprint) https://doi.org/10.1155/2015/576093

[22] Abramowitz, M. and Stegun, I.A. (1965) Handbook of Mathematical Functions with Formulas, Graphs, and Mathematical Tables. Dover, New York.

[23] von Seggern, D. (1992) CRC Standard Curves and Surfaces. CRC Press, New York.

[24] Thompson, W.J. (1997) Atlas for Computing Mathematical Functions. WileyInterscience, New York.

[25] Gradshteyn, I.S., Ryzhik, I.M., Jeffrey, A. and Zwillinger, D. (2007) Table of Integrals, Series, and Products. Academic Press, New York.

[26] Olver, F.W.J., Lozier, D.W., Boisvert, R.F. and Clark, C.W. (2010) NIST Handbook of Mathematical Functions. Cambridge University Press, Cambridge.

[27] Zaninetti, L. (2019) The Distance Modulus in Dark Energy and Cardassian Cosmologies via the Hypergeometric Function International Journal of Astronomy and Astrophysics, 9, 231-246. (Preprint) https://doi.org/10.4236/ijaa.2019.93017

[28] Freese, K. and Lewis, M. (2002) Cardassian Expansion: A Model in Which the Universe Is Flat, Matter Dominated, and Accelerating. Physics Letters B, 540, 1-8. (Preprint) https://doi.org/10.1016/S0370-2693(02)02122-6

[29] Freese, K. (2003) Generalized Cardassian Expansion: A Model in Which the Universe Is Flat, Matter Dominated, and Accelerating. Nuclear Physics B: Proceedings Supplements, 124, 50-54. (Preprint) https://doi.org/10.1016/S0920-5632(03)02076-0

[30] Baes, M., Camps, P. and Van De Putte, D. (2017) Analytical Expressions and Numerical Evaluation of the Luminosity Distance in a Flat Cosmology. Monthly Notices of the Royal Astronomical Society, 468, 927-930. (Preprint) https://doi.org/10.1093/mnras/stx537

[31] Zaninetti, L. (2019) A New Analytical Solution for the Distance Modulus in Flat Cosmology. International Journal of Astronomy and Astrophysics, 9, 51-62. (Preprint)

[32] Starobinsky, A.A. (1980) A New Type of Isotropic Cosmological Models without 
Singularity. Physics Letters B, 91, 99-102.

https://doi.org/10.1016/0370-2693(80)90670-X

[33] Guth, A.H. and Weinberg, E.J. (1981) Cosmological Consequences of a First-Order Phase Transition in the $\mathrm{SU}_{5}$ Grand Unified Model. Physical Review D, 23, 876-885. https://doi.org/10.1103/PhysRevD.23.876

[34] Ratra, B. and Peebles, P.J.E. (1988) Cosmological Consequences of a Rolling Homogeneous Scalar Field. Physical Review D, 37, 3406-3427. https://doi.org/10.1103/PhysRevD.37.3406

[35] Steinhardt, P.J. and Caldwell, R.R. (1998) Introduction to Quintessence. In: Byun, Y.I. and Ng, K.W., Eds, Cosmic Microwave Background and Large Scale Structure of the Universe, Vol. 151, Astronomical Society of the Pacific, San Francisco, 13.

[36] Avsajanishvili, O., Huang, Y., Samushia, L. and Kahniashvili, T. (2018) The Observational Constraints on the Flat $\varphi \mathrm{CDM}$ Models. European Physical Journal C, 78, Article No. 773. (Preprint) https://doi.org/10.1140/epjc/s10052-018-6233-y

[37] Mamon, A.A., Bamba, K. and Das, S. (2017) Constraints on Reconstructed Dark Energy Model from SN Ia and BAO/CMB Observations. European Physical Journal C, 77, Article No. 29. (Preprint) https://doi.org/10.1140/epjc/s10052-016-4590-y

[38] Einstein, A. and de Sitter, W. (1932) On the Relation between the Expansion and the Mean Density of the Universe. Proceedings of the National Academy of Sciences of the United States of America, 18, 213-214. https://doi.org/10.1073/pnas.18.3.213

[39] Krisciunas, K. (1993) Look-Back Time the Age of the Universe and the Case for a Positive Cosmological Constant. Journal of the Royal Astronomical Society of Canada, 87, Article No. 223. (Preprint)

[40] Ryden, B. (2003) Introduction to Cosmology. Addison Wesley, San Francisco, CA.

[41] Lang, K. (2013) Astrophysical Formulae: Space, Time, Matter and Cosmology Astronomy and Astrophysics Library. Springer, Berlin.

[42] Heymann, Y. (2013) On the Luminosity Distance and the Hubble Constant. Progress in Physics, 3, 5-6.

[43] Milne, E.A. (1933) World-Structure and the Expansion of the Universe. Zeitschrift fur Astrophysik, 6, 1.

[44] Chodorowski, M.J. (2005) Cosmology under Milne's Shadow. Publications of the Astronomical Society of Australia, 22, 287-291. (Preprint)

https://doi.org/10.1071/AS05016

[45] Adamek, J., Di Dio, E., Durrer, R. and Kunz, M. (2014) Distance-Redshift Relation in Plane Symmetric Universes. Physical Review D, 89, Article ID: 063543. (Preprint) https://doi.org/10.1103/PhysRevD.89.063543

[46] Brynjolfsson, A. (2004) Redshift of Photons Penetrating a Hot Plasma. arXiv:astro$\mathrm{ph} / 0401420$

[47] Brynjolfsson, A. (2006) Magnitude-Redshift Relation for SNe Ia, Time Dilation, and Plasma Redshift. ArXiv:astro-ph/0602500

[48] Marmet, L. (2018) On the Interpretation of Spectral Red-Shift in Astrophysics: A Survey of Red-Shift Mechanisms-II. arXiv e-prints arXiv:1801.07582. (Preprint)

[49] Zaninetti, L. (2015) On the Number of Galaxies at High Redshift. Galaxies, 3, 129155. https://doi.org/10.3390/galaxies3030129

[50] Press, W.H., Teukolsky, S.A., Vetterling, W.T. and Flannery, B.P. (1992) Numerical Recipes in FORTRAN. The Art of Scientific Computing. Cambridge University Press, Cambridge.

[51] Akaike, H. (1974) A New Look at the Statistical Model Identification. IEEE Trans- 
actions on Automatic Control, 19, 716-123. https://doi.org/10.1109/TAC.1974.1100705

[52] Liddle, A.R. (2004) How Many Cosmological Parameters? Monthly Notices of the Royal Astronomical Society, 351, L49-L53. https://doi.org/10.1111/j.1365-2966.2004.08033.x

[53] Godlowski, W. and Szydowski, M. (2005) Constraints on Dark Energy Models from Supernovae. In: Turatto, M., Benetti, S., Zampieri, L. and Shea, W., Eds, 1604-2004: Supernovae as Cosmological Lighthouses, Vol. 342, Astronomical Society of the Pacific, San Francisco, 508-516

[54] Bevington, P.R. and Robinson, D.K. (2003) Data Reduction and Error Analysis for the Physical Sciences. McGraw-Hill, New York.

[55] Wei, H. (2010) Observational Constraints on Cosmological Models with the Updated Long Gamma-Ray Bursts Journal of Cosmology and Astroparticle Physic, 8 , Article No. 020. (Preprint) https://doi.org/10.1088/1475-7516/2010/08/020

[56] Etherington, I.M.H. (1933) On the Definition of Distance in General Relativity. Philosophical Magazine, 15, 761-773. https://doi.org/10.1080/14786443309462220

[57] Remez, E. (1934) Sur la détermination des polynômes d' approximation de degré donnée. Communications of Kharkov Mathematical Society, 10, Article No. 41

[58] Remez, E. (1957) General Computation Methods of Chebyshev Approximation. The Problems with Linear Real Parameters. Publishing House of the Academy of Science of the Ukrainian SSR, Kiev.

[59] Braatz, J.A., Reid, M.J., Humphreys, E.M.L., Henkel, C., Condon, J.J. and Lo, K.Y. (2010) The Megamaser Cosmology Project. II. The Angular-Diameter Distance to UGC 3789. The Astrophysical Journal, 718, 657-665. (Preprint) https://doi.org/10.1088/0004-637X/718/2/657

[60] Kuo, C.Y., Braatz, J.A., Reid, M.J., Lo, K.Y., Condon, J.J., Impellizzeri, C.M.V. and Henkel, C. (2013) The Megamaser Cosmology Project. V. An Angular-diameter Distance to NGC 6264 at 140 Mpc. The Astrophysical Journal, 767, Article No. 155. (Preprint) https://doi.org/10.1088/0004-637X/767/2/155

[61] Melia, F. and Yennapureddy, M.K. (2018) The Maximum Angular-Diameter Distance in Cosmology. Monthly Notices of the Royal Astronomical Society, 480, 2144-2152. (Preprint) https://doi.org/10.1093/mnras/sty1962

[62] Eales, S., Dunne, L., Clements, D., Cooray, A., De Zotti, G., Dye, S., et al. (2010) The Herschel ATLAS. Publications of the Astronomical Society of Pacific, 122, 499-515. (Preprint)

[63] Tamura, Y., Oguri, M., Iono, D., Hatsukade, B., Matsuda, Y. and Hayashi, M. (2015) High-Resolution ALMA Observations of SDP.81. I. The Innermost Mass Profile of the Lensing Elliptical Galaxy Probed by 30 Milli-Arcsecond Images. Publications of the Astronomical Society of Japan, 67, 72. (Preprint) https://doi.org/10.1093/pasj/psv040

[64] ALMA Partnership (2015) The 2014 ALMA Long Baseline Campaign: Observations of the Strongly Lensed Submillimeter Galaxy HATLAS J090311.6+003906 at z=3.042. The Astrophysical Journal Letters, 808, Article No, L4. (Preprint) https://doi.org/10.1088/2041-8205/808/1/L4

[65] Rybak, M., Vegetti, S., McKean, J.P., Andreani, P. and White, S.D.M. (2015) ALMA Imaging of SDP.81-II. A Pixelated Reconstruction of the CO Emission Lines. Monthly Notices of the Royal Astronomical Society: Letters, 453, L26-L30. (Preprint) https://doi.org/10.1093/mnrasl/slv092

[66] Hatsukade, B., Tamura, Y., Iono, D., Matsuda, Y., Hayashi, M. and Oguri, M. (2015) 
High-Resolution ALMA Observations of SDP.81. II. Molecular Clump Properties of a Lensed Submillimeter Galaxy at $\mathrm{z}=3.042$. Publications of the Astronomical Society of Japan, 67, Article No. 93. (Preprint)

[67] Wong, K.C., Suyu, S.H. and Matsushita, S. (2015) The Innermost Mass Distribution of the Gravitational Lens SDP.81 from ALMA Observations. The Astrophysical Journal, 811, Article No. 115. (Preprint) https://doi.org/10.1088/0004-637X/811/2/115

[68] Hezaveh, Y.D., Dalal, N. and Marrone, D.P. (2016) Detection of Lensing Substructure Using ALMA Observations of the Dusty Galaxy SDP.81. The Astrophysical Journal, 823, Article No. 37 (Preprint) https://doi.org/10.3847/0004-637X/823/1/37

[69] Zaninetti, L. (2017) The Ring Produced by an Extra-Galactic Superbubble in Flat Cosmology. Journal of High Energy Physics, Gravitation and Cosmology, 3, 339-359. https://doi.org/10.4236/jhepgc.2017.32029

[70] Mohr, P.J., Taylor, B.N. and Newell, D.B. (2012) CODATA Recommended Values of the Fundamental Physical Constants: 2010. Reviews of Modern Physics, 84, 1527 1605. https://doi.org/10.1103/RevModPhys.84.1527

[71] Padé, H. (1892) Sur la représentation approchée d'une fonction par des fractions rationnelles. Annales scientifiques de l'École Normale Supérieure, 9, 3-93. https://doi.org/10.24033/asens.378

[72] Baker, G. (1975) Essentials of Padé approximants. Academic Press, New York.

[73] Baker, G.A. and Graves-Morris, P.R. (1996) Padé approximants. Vol. 59, Cambridge University Press, Cambridge. 


\section{Appendix A. The Padé Approximant}

Given a function $f(z)$, the Padé approximant, after [71], is:

$$
f(z)=\frac{a_{0}+a_{1} z+\cdots+a_{p} z^{p}}{b_{0}+b_{1} z+\cdots+b_{q} z^{q}},
$$

where the notation is the same as in [26]

The coefficients $a_{i}$ and $b_{i}$ are found through Wynn's cross rule, see [72] [73] and our choice is $p=2$ and $q=2$. The choice of $p$ and $q$ is a compromise between precision (associated with high values for $p$ and $q$ ) and the simplicity of the expressions to manage (associated with low values for $p$ and $q$ ). The argument of the integral to be done is the inverse of $E(z)$, see Equation (6),

$$
\frac{1}{E(z)}=\frac{1}{\sqrt{\Omega_{\mathrm{M}}(1+z)^{3}+\Omega_{K}(1+z)^{2}+\Omega_{\Lambda}}},
$$

and the Padé approximant is

$$
\frac{1}{E(z)}=\frac{a_{0}+a_{1} z+a_{2} z^{2}}{b_{0}+b_{1} z+b_{2} z^{2}},
$$

where,

$$
\begin{aligned}
a_{0}= & 16\left(32 \Omega_{K}^{3} \Omega_{\Lambda}+16 \Omega_{K}^{2} \Omega_{\Lambda}^{2}+160 \Omega_{K}^{2} \Omega_{\Lambda} \Omega_{\mathrm{M}}+24 \Omega_{K}^{2} \Omega_{\mathrm{M}}^{2}\right. \\
& +64 \Omega_{K} \Omega_{\Lambda}^{2} \Omega_{\mathrm{M}}+320 \Omega_{K} \Omega_{\Lambda} \Omega_{\mathrm{M}}^{2}+40 \Omega_{K} \Omega_{\mathrm{M}}^{3}+96 \Omega_{\Lambda}^{2} \Omega_{\mathrm{M}}^{2} \\
& \left.+192 \Omega_{\Lambda} \Omega_{\mathrm{M}}^{3}+15 \Omega_{\mathrm{M}}^{4}\right)\left(\Omega_{\mathrm{M}}+\Omega_{K}+\Omega_{\Lambda}\right)^{4} \\
a_{1}= & 4\left(128 \Omega_{K}^{4} \Omega_{\Lambda}+32 \Omega_{K}^{3} \Omega_{\Lambda}^{2}+704 \Omega_{K}^{3} \Omega_{\Lambda} \Omega_{\mathrm{M}}-16 \Omega_{K}^{2} \Omega_{\Lambda}^{2} \Omega_{\mathrm{M}}\right. \\
& +1456 \Omega_{K}^{2} \Omega_{\Lambda} \Omega_{\mathrm{M}}^{2}+32 \Omega_{K}^{2} \Omega_{\mathrm{M}}^{3}-64 \Omega_{K} \Omega_{\Lambda}^{3} \Omega_{\mathrm{M}}-384 \Omega_{K} \Omega_{\Lambda}^{2} \Omega_{\mathrm{M}}^{2} \\
& +1512 \Omega_{K} \Omega_{\Lambda} \Omega_{\mathrm{M}}^{3}+50 \Omega_{K} \Omega_{\mathrm{M}}^{4}-192 \Omega_{\Lambda}^{3} \Omega_{\mathrm{M}}^{2}-288 \Omega_{\Lambda}^{2} \Omega_{\mathrm{M}}^{3} \\
& \left.+648 \Omega_{\Lambda} \Omega_{\mathrm{M}}^{4}+15 \Omega_{\mathrm{M}}^{5}\right)\left(\Omega_{\mathrm{M}}+\Omega_{K}+\Omega_{\Lambda}\right)^{3} \\
a_{2}= & \left(256 \Omega_{K}^{4} \Omega_{\Lambda} \Omega_{\mathrm{M}}-64 \Omega_{K}^{3} \Omega_{\Lambda}^{3}+320 \Omega_{K}^{3} \Omega_{\Lambda}^{2} \Omega_{\mathrm{M}}+960 \Omega_{K}^{3} \Omega_{\Lambda} \Omega_{\mathrm{M}}^{2}\right. \\
- & 320 \Omega_{K}^{2} \Omega_{\Lambda}^{3} \Omega_{\mathrm{M}}+240 \Omega_{K}^{2} \Omega_{\Lambda}^{2} \Omega_{\mathrm{M}}^{2}+1440 \Omega_{K}^{2} \Omega_{\Lambda} \Omega_{\mathrm{M}}^{3}+16 \Omega_{K}^{2} \Omega_{\mathrm{M}}^{4} \\
- & 1600 \Omega_{K} \Omega_{\Lambda}^{3} \Omega_{\mathrm{M}}^{2}-480 \Omega_{K} \Omega_{\Lambda}^{2} \Omega_{\mathrm{M}}^{3}+1140 \Omega_{K} \Omega_{\Lambda} \Omega_{\mathrm{M}}^{4}+20 \Omega_{K} \Omega_{\mathrm{M}}^{5} \\
- & 256 \Omega_{\Lambda}^{4} \Omega_{\mathrm{M}}^{2}-1600 \Omega_{\Lambda}^{3} \Omega_{\mathrm{M}}^{3}-240 \Omega_{\Lambda}^{2} \Omega_{\mathrm{M}}^{4}+380 \Omega_{\Lambda} \Omega_{\mathrm{M}}^{5} \\
+ & \left.5 \Omega_{\mathrm{M}}^{6}\right)\left(\Omega_{\mathrm{M}}+\Omega_{K}+\Omega_{\Lambda}\right)^{2} \\
b_{1}= & 16\left(\Omega_{\mathrm{M}}+\Omega_{K}+\Omega_{\Lambda}\right)^{9 / 2}\left(32 \Omega_{K}^{3} \Omega_{\Lambda}+16 \Omega_{K}^{2} \Omega_{\Lambda}^{2}+160 \Omega_{K}^{2} \Omega_{\Lambda} \Omega_{\mathrm{M}}\right. \\
+ & 96 \Omega_{K}^{3} \Omega_{\mathrm{M}}^{2}+336 \Omega_{K}^{2} \Omega_{\Lambda}^{2} \Omega_{\mathrm{M}}+3696 \Omega_{K}^{2} \Omega_{\Lambda} \Omega_{\mathrm{M}}^{2}+336 \Omega_{K}^{2} \Omega_{\mathrm{M}}^{3} \\
- & 64 \Omega_{K} \Omega_{\Lambda}^{3} \Omega_{\mathrm{M}}+384 \Omega_{K} \Omega_{\Lambda}^{2} \Omega_{\mathrm{M}}^{2}+4200 \Omega_{K} \Omega_{\Lambda} \Omega_{\mathrm{M}}^{3}+350 \Omega_{K} \Omega_{\mathrm{M}}^{4} \\
+ & \left.192 \Omega_{\Lambda}^{3} \Omega_{\mathrm{M}}^{2}+288 \Omega_{\Lambda}^{2} \Omega_{\mathrm{M}}^{3}+1800 \Omega_{\Lambda} \Omega_{\mathrm{M}}^{4}+105 \Omega_{\mathrm{M}}^{5}\right) \\
+ & 24 \Omega_{K}^{2} \Omega_{\mathrm{M}}^{2}+64 \Omega_{K} \Omega_{\Lambda}^{2} \Omega_{\mathrm{M}}+320 \Omega_{K} \Omega_{\Lambda} \Omega_{\mathrm{M}}^{2}+40 \Omega_{K} \Omega_{\mathrm{M}}^{3} \\
+ & \left.96 \Omega_{\Lambda}^{2} \Omega_{\mathrm{M}}^{2}+192 \Omega_{\Lambda} \Omega_{\mathrm{M}}^{3}+15 \Omega_{\mathrm{M}}^{4}\right) \\
\left.b_{0}\right) &
\end{aligned}
$$




$$
\begin{aligned}
b_{2}= & \left(\Omega_{\mathrm{M}}+\Omega_{K}+\Omega_{\Lambda}\right)^{5 / 2}\left(512 \Omega_{K}^{5} \Omega_{\Lambda}+384 \Omega_{K}^{4} \Omega_{\Lambda}^{2}+3584 \Omega_{K}^{4} \Omega_{\Lambda} \Omega_{\mathrm{M}}\right. \\
& +192 \Omega_{K}^{3} \Omega_{\Lambda}^{3}+1984 \Omega_{K}^{3} \Omega_{\Lambda}^{2} \Omega_{\mathrm{M}}+10752 \Omega_{K}^{3} \Omega_{\Lambda} \Omega_{\mathrm{M}}^{2}+320 \Omega_{K}^{3} \Omega_{\mathrm{M}}^{3} \\
& +960 \Omega_{K}^{2} \Omega_{\Lambda}^{3} \Omega_{\mathrm{M}}+5136 \Omega_{K}^{2} \Omega_{\Lambda}^{2} \Omega_{\mathrm{M}}^{2}+17760 \Omega_{K}^{2} \Omega_{\Lambda} \Omega_{\mathrm{M}}^{3}+840 \Omega_{K}^{2} \Omega_{\mathrm{M}}^{4} \\
& +2752 \Omega_{K} \Omega_{\Lambda}^{3} \Omega_{\mathrm{M}}^{2}+7392 \Omega_{K} \Omega_{\Lambda}^{2} \Omega_{\mathrm{M}}^{3}+15060 \Omega_{K} \Omega_{\Lambda} \Omega_{\mathrm{M}}^{4}+700 \Omega_{K} \Omega_{\mathrm{M}}^{5} \\
& \left.+256 \Omega_{\Lambda}^{4} \Omega_{\mathrm{M}}^{2}+2752 \Omega_{\Lambda}^{3} \Omega_{\mathrm{M}}^{3}+3696 \Omega_{\Lambda}^{2} \Omega_{\mathrm{M}}^{4}+5020 \Omega_{\Lambda} \Omega_{\mathrm{M}}^{5}+175 \Omega_{\mathrm{M}}^{6}\right)
\end{aligned}
$$

The indefinite integral of (3), $F_{2,2}$, is:

$$
\begin{aligned}
& F_{2,2}\left(z ; a_{0}, a_{1}, a_{2}, b_{0}, b_{1}, b_{2}\right) \\
& =\frac{a_{2} z}{b_{2}}+\frac{1}{2} \frac{\ln \left(z^{2} b_{2}+z b_{1}+b_{0}\right) a_{1}}{b_{2}}-\frac{1}{2} \frac{\ln \left(z^{2} b_{2}+z b_{1}+b_{0}\right) a_{2} b_{1}}{b_{2}^{2}} \\
& +2 \frac{a_{0}}{\sqrt{4 b_{0} b_{2}-b_{1}^{2}}} \arctan \left(\frac{2 z b_{2}+b_{1}}{\sqrt{4 b_{0} b_{2}-b_{1}^{2}}}\right) \\
& -2 \frac{a_{2} b_{0}}{b_{2} \sqrt{4 b_{0} b_{2}-b_{1}^{2}}} \arctan \left(\frac{2 z b_{2}+b_{1}}{\sqrt{4 b_{0} b_{2}-b_{1}^{2}}}\right) \\
& -\frac{b_{1} a_{1}}{b_{2} \sqrt{4 b_{0} b_{2}-b_{1}^{2}}} \arctan \left(\frac{2 z b_{2}+b_{1}}{\sqrt{4 b_{0} b_{2}-b_{1}^{2}}}\right) \\
& +\frac{b_{1}^{2} a_{2}}{b_{2}^{2} \sqrt{4 b_{0} b_{2}-b_{1}^{2}}} \arctan \left(\frac{2 z b_{2}+b_{1}}{\sqrt{4 b_{0} b_{2}-b_{1}^{2}}}\right) .
\end{aligned}
$$

